1. This work has not yet been peer-reviewed and is provided by the contributing author(s) as a means 2 to ensure timely dissemination of scholarly and technical work on a noncommercial basis. Copy${ }_{3}$ right and all rights therein are maintained by the author(s) or by other copyright owners. It is ${ }_{4}$ understood that all persons copying this information will adhere to the terms and constraints in${ }_{5}$ voked by each author's copyright. This work may not be reposted without explicit permission of 6 the copyright owner.

7 This work has been submitted to the Journal of Physical Oceanography. Copyright in this work s may be transferred without further notice.

\title{
Centrifugal and symmetric instability during Ekman adjustment of the
}

\section{bottom boundary layer}

Department of Atmospheric and Oceanic Science, University of Maryland, College Park

${ }_{15}^{*}$ Corresponding author address: Dept. of Atmospheric and Oceanic Sciences, University of Mary-

16 land, 4254 Stadium Dr., College Park, MD, 20742.

17 E-mail: wenegrat@umd.edu 
ABSTRACT

Flow along isobaths of a sloping lower boundary generates an across-isobath

19 Ekman transport in the bottom boundary layer. When this Ekman transport is down the slope it causes convective mixing - much like a downfront wind in ${ }_{21}$ the surface boundary layer - destroying stratification and potential vorticity. ${ }_{22}$ In this manuscript we show how this can lead to the development of a forced ${ }_{23}$ centrifugal or symmetric instability regime, where the potential vorticity flux ${ }_{24}$ generated by friction along the boundary is balanced by submesoscale insta${ }_{25}$ bilities that return the boundary layer potential vorticity to zero. This balance provides a strong constraint on the boundary layer evolution, which we use ${ }_{27}$ to develop theory that explains the evolution of the boundary layer thickness, ${ }_{28}$ the rate at which the instabilities extract energy from the geostrophic flow ${ }_{29}$ field, and the magnitude and vertical structure of the dissipation. Finally, we so show using theory and a high-resolution numerical model how the presence of ${ }_{31}$ centrifugal or symmetric instabilities alters the time-dependent Ekman adjust32 ment of the boundary layer, delaying Ekman buoyancy arrest and enhancing зз the total energy removed from the balanced flow field. Submesoscale insta${ }_{34}$ bilities of the bottom boundary layer may therefore play an important, largely ${ }_{35}$ overlooked, role in the energetics of flow over topography in the ocean. 


\section{Introduction}

The ocean bottom boundary layer (BBL) over sloping topography often has a structure reminiscent of a surface mixed layer front, with isopycnals that slope downward from the interior towards the topography (figure 1). One way that this frontal BBL structure can develop is when interior flow along isobaths of a sloping lower boundary forces an across-isobath bottom Ekman transport (MacCready and Rhines 1991). This Ekman transport follows the sloping lower boundary, which crosses isopycnals whenever the interior is stratified, thereby generating an advective flux of buoyancy. When the transport is towards deeper water (downslope), the advective buoyancy flux brings buoyant water down along the bottom, leading to convective mixing, which on the slope acts to increase the horizontal buoyancy gradient at the expense of the vertical gradient.

The case of downslope Ekman transport is therefore closely analogous to the case of a downfront wind stress (Thomas 2005; Thomas and Ferrari 2008), where a wind aligned with a frontal jet drives an Ekman transport that is directed from the dense side to the light side of a surface ocean front. This Ekman buoyancy flux has been shown to modify the surface boundary layer in a wide variety of ways, one of the most consequential of which is through the generation of symmetric instability (SI), a fast growing submesoscale instability associated with 2D overturning circulations in the cross-front plane (Stone 1966; Haine and Marshall 1998). A partial list of the aspects of the surface boundary layer evolution which SI is known to affect includes the rates of: mixed-layer deepening, entrainment, restratification, kinetic energy dissipation, and buoyancy mixing (Taylor and Ferrari 2010; D’Asaro et al. 2011; Thomas et al. 2013, 2016).

Several lines of evidence point to the existence of similar processes in the BBL, starting with theoretical and modeling work by Allen and Newberger (1998), who noted that when the BBL is in thermal wind balance (the 'arrested' Ekman layer, Garrett et al. 1993) it is unstable to grow- 
ing symmetric modes, suggesting the incompleteness of 1D theory. Using 2D simulations they investigated the finite-amplitude behavior of SI, arguing that instabilities are likely found both in response to Ekman adjustment of the boundary layer to an interior flow and in response to downwelling favorable surface winds (Allen and Newberger 1993, 1998). More recent idealized 3D numerical simulations of a tidal mixing front (Brink and Cherian 2013), and dense shelf overflows (Yankovsky and Legg 2019), likewise indicate the presence of both SI and baroclinic modes, consistent with the predictions of Wenegrat et al. (2018). Finally, perhaps the most compelling evidence currently available comes from recent observations taken in the Southern Ocean, which showed that downslope Ekman flows in the deep ocean, generated by the Antarctic bottom water flowing along steep topography, led to conditions conducive to symmetric and centrifugal instabilities (CI, Naveira Garabato et al. 2019). These conditions were also associated with enhanced turbulent dissipation rates (Naveira Garabato et al. 2019), similar to observations of SI in the surface boundary layer (D’Asaro et al. 2011).

The primary goal of this paper is therefore to examine centrifugal and symmetric instability in the BBL in the case where a steady interior flow over uniformly sloping topography drives a downslope Ekman transport. We focus on the time-dependent adjustment process, and the development of a 'forced' regime where downslope Ekman buoyancy fluxes maintain persistent SI/CI. The similarity between downslope and across-front wind-driven Ekman transports is used to adapt the insightful derivations provided in Taylor and Ferrari (2010, hereinafter TF10) for the surface boundary layer to the case of a BBL over sloping topography. This allows us to extend earlier work on this topic to provide a theoretical framework that explains many aspects of the BBL evolution in the presence of SI and CI, including how the boundary layer height and stratification evolve, the rate at which the instabilities extract energy from the mean flow, and the magnitude and vertical structure of the turbulent dissipation. 
The manuscript is organized as follows. In section 2 we introduce the high-resolution numerical model we use to test the theory, and provide a brief qualitative discussion of the evolution of two representative simulations. In section 3 we develop the theory of the BBL evolution in the presence of SI/CI, and test the predictions against the numerical simulations. In section 4 we show how SI/CI modifies the energetics of the BBL and provide simple scalings for the turbulent dissipation that reproduce the numerical results. Finally, in section 5 we discuss how SI/CI modifies the classical 1D conception of the Ekman adjustment of the BBL.

\section{Numerical Simulations}

\section{a. Numerical model configuration}

To explore the role of instabilities during Ekman adjustment of the BBL we perform highresolution numerical simulations of a stratified flow oriented along isobaths of a sloping bottom. The domain setup is idealized, assuming uniform topographic slope $(\theta)$, periodicity in the along and across isobath directions, a steady barotropic interior flow $\left(V_{\infty}\right)$, and uniform interior stratification $\left(N_{\infty}^{2}\right.$, figure 1). Our interest is in the SI/CI modes, hence we only consider the case where the interior flow generates downwelling in the bottom Ekman layer (ie. $V_{\infty}>0$ in the Northern Hemisphere for the geometry shown in figure 1).

It is useful to work in a coordinate system rotated to align with the sloping bottom (figure 1), where $x$ is the across-isobath (across-slope) direction, $y$ is the along-isobath (along-slope) direction, and $z$ is the slope-normal direction (defined such that the bottom is at $z=0$ ). When coordinates are referenced in the standard, non-rotated, coordinate system they will be indicated using a carat (ie. $\hat{z}$ aligns with the direction of gravity). Separating the total velocity and buoyancy fields into interior (denoted with subscript $\infty$ ) and perturbation quantities (denoted by lowercase 
variables), such that $\mathbf{u}_{\mathbf{T}}=\left(u, v+V_{\infty}, w\right)$, the equations governing the perturbations are (Wenegrat et al. 2018),

$$
\begin{aligned}
\frac{\partial u}{\partial t}+\mathbf{u}_{\mathbf{T}} \cdot \nabla u-f \cos \theta v & =-\frac{1}{\rho_{o}} \frac{\partial p}{\partial x}+b \sin \theta+v \nabla^{2} u \\
\frac{\partial v}{\partial t}+\mathbf{u}_{\mathbf{T}} \cdot \nabla v-f \sin \theta w+f \cos \theta u & =-\frac{1}{\rho_{o}} \frac{\partial p}{\partial y}+v \nabla^{2} v \\
\frac{\partial w}{\partial t}+\mathbf{u}_{\mathbf{T}} \cdot \nabla w+f \sin \theta v & =-\frac{1}{\rho_{o}} \frac{\partial p}{\partial z}+b \cos \theta+v \nabla^{2} w \\
\frac{\partial b}{\partial t}+\mathbf{u}_{\mathbf{T}} \cdot \nabla b+u N_{\infty}^{2} \sin \theta+w N_{\infty}^{2} \cos \theta & =\kappa \nabla^{2} b \\
\nabla \cdot \mathbf{u} & =0 .
\end{aligned}
$$

Note that the use of periodic boundary conditions in the across-slope $(x)$ direction requires that the mean across-slope buoyancy gradient remains fixed in time, with magnitude $N_{\infty}^{2} \sin \theta$. This setup is therefore similar to the 'frontal-zone' configuration commonly used in spectral simulations of surface boundary layer fronts, where a fixed magnitude horizontal buoyancy gradient is imposed (eg. Taylor and Ferrari 2010; Thomas and Taylor 2010). Importantly however, in the BBL case both the mean horizontal buoyancy gradient, and the mean vertical vorticity, are free to evolve in time.

Bottom boundary conditions are given by,

$$
u=0, \quad v+V_{\infty}=0, \quad w=0, \quad \frac{\partial b}{\partial z}+N_{\infty}^{2} \cos \theta=0, \quad \text { at } z=0
$$

These equations are solved numerically using the pseudo-spectral code Dedalus (Burns et al. $2016,2019)$ in a 2D domain $(x-z)$ that is periodic in the across and along-isobath directions $(x$ and $y$ ), and bounded by rigid walls in the slope-normal direction $(z)$. The 2D domain allows for computationally efficient exploration of the 2D SI/CI overturning instabilities, but will suppress the emergence of 3D baroclinic modes expected after a transient SI phase in cases with low interior slope Burger number, $S_{\infty}=N_{\infty} \theta / f$ (Brink and Cherian 2013; Wenegrat et al. 2018). In regions 
with large slope Burger number topographic suppression of the baroclinic growth rates allows for persistent SI/CI even in 3D simulations (Wenegrat et al. 2018).

In all simulations the effective resolution after de-aliasing is $\Delta x=1 \mathrm{~m}$ and $\Delta z=0.01-1.2 \mathrm{~m}$, with enhanced resolution near the lower and upper-boundaries. The domain size is $1 \mathrm{~km}$ in the across-slope $(x)$ direction and $200 \mathrm{~m}$ in the slope-normal $(z)$ direction, except where larger domains were determined to be necessary to fully resolve the instabilities and boundary layer evolution (as indicated in table 1). A sponge region with Rayleigh damping of perturbations is applied in the upper $20 \mathrm{~m}$ of the domain to reduce wave reflection (as in TF10). A constant viscosity and diffusivity of $v=\kappa=10^{-4} \mathrm{~m}^{2} \mathrm{~s}^{-1}$ are used, again consistent with TF10, giving a laminar Ekman layer depth of $\delta_{e}=\sqrt{2 \mathrm{v} / f}=1.4 \mathrm{~m}$. The near-wall viscous sublayer is confirmed to be resolved with at least 2 grid points within one viscous wall unit of the boundary at all times $\left(\delta_{v}=v / u^{*}\right.$, where $u^{*}=\sqrt{|\boldsymbol{\tau}| / \rho_{o}}$, and $|\boldsymbol{\tau}|$ is the magnitude of the bottom stress).

\section{b. Description of simulation evolution}

The full set of simulations considered here span a wide-range of slope angles, interior stratification, and slope Burger numbers (as listed in table 1). It is however useful to begin with a brief qualitative description of several representative simulations. Figure 2 shows the evolution of simulation SI-1, which has an initial slope Burger number of $S_{\infty}=0.6$, indicating a moderately steep-regime where symmetric instability is expected (Wenegrat et al. 2018). The simulation begins with a barotropic interior flow along the slope $\left(V_{\infty}\right)$, which generates a downslope Ekman flow $(u<0)$ within approximately an inertial period in response to the associated along-slope bottom stress. This Ekman flow advects buoyant water down the slope in a thin near boundary Ekman layer, which generates convective mixing that destroys stratification, producing a bottom boundary layer which grows to $\sim 50 \mathrm{~m}$ thickness after 15 days. 
The destruction of stratification by the downslope Ekman transport also leads to a boundary flux of Ertel potential vorticity (PV, Benthuysen and Thomas 2012), defined as $q=\boldsymbol{\omega} \cdot \nabla(b+$ $\left.N_{\infty}^{2} \cos \theta z+N_{\infty}^{2} \sin \theta x\right)$, where $\omega$ is the total absolute vorticity vector, and the gradient operator is in the rotated coordinate system. This leads to a BBL characterized by $f q<0$ (which can be seen in the first several days of the simulation), and $f(f+\partial v / \partial \hat{x})>0$, a state which is unstable to symmetric instability (Haine and Marshall 1998; Thomas et al. 2013). In classic 1D theory, or in a simulation where submesoscale instabilities were not resolved, this evolution would continue, with convective turbulence deepening the well-mixed boundary layer until an arrested Ekman state was achieved, or the flow relaminarized (MacCready and Rhines 1991, 1993; Ruan et al. 2019). Here however the state of $f q<0$ gives rise to rapidly growing symmetric instability (figure 3 ), which reaches finite amplitude within several days, and returns the boundary layer to $q \approx 0$ (figure 2). Also evident in figure 3 are secondary Kelvin-Helmholtz instabilities, generated by the sheared SI overturning cells, which enhance the boundary layer dissipation (Taylor and Ferrari 2009, and section 4). These conditions, where an Ekman buoyancy flux pushes the boundary layer towards $f q<0$ and symmetric instabilities return the boundary layer to the point of marginal stability, $q \approx$ 0 , is a regime known from the surface boundary layer literature as 'forced symmetric instability' (Taylor and Ferrari 2010; Thomas and Taylor 2010; Thomas et al. 2013), newly identified here as a feature of the BBL.

A useful diagnostic for determining the fastest growing instability type in each portion of the domain comes from linear theory, developed in Thomas et al. (2013). Assuming a flow that is in approximate geostrophic balance, an instability angle can be defined as $\phi_{R i_{b}}=$ $\tan ^{-1}\left(-|\partial b / \partial \hat{x}|^{2} / f^{2} N^{2}\right)$, such that growing instabilities will occur when $\phi_{R i_{b}}$ is smaller than a critical angle of $\phi_{c}=\tan ^{-1}\left(-\left(f+\partial v_{g} / \partial \hat{x}\right) / f\right)$ (Thomas et al. 2013). Symmetric modes dominate for $-90<\phi_{R i_{b}}<-45$, growing through vertical shear production, and when $-45<\phi_{R i_{b}}<\phi_{c}$ 
mixed symmetric-centrifugal modes grow via both lateral and vertical shear production. When the stratification becomes unstable $\left(N^{2}<0, \phi_{b}<-90\right)$, the fastest growing mode will either be a gravitational instability or a mixed gravitational-symmetric mode depending on the relative magnitude of the vertical buoyancy production and shear production (see Thomas et al. 2013, appendix). To reduce noise associated with calculating these quantities from the high-resolution numerical model we first smooth the stratification and buoyancy gradients to $\Delta x \approx 14 \mathrm{~m}$ and $\Delta z \approx 3 \mathrm{~m}$ resolution, and use across-slope averaged profiles of the geostrophic vertical relative vorticity to determine $\phi_{c}$ and the transition between gravitational and mixed gravitational-symmetric modes (see equation 41 of Thomas et al. 2013). The resulting estimate of $\phi_{R i_{b}}$ for simulation SI-1 is shown in figure 4, with the color scale indicating the primary instability type, illustrating how most of the BBL is dominated by symmetric instability. Near the lower-boundary regions of gravitational and mixed gravitational-symmetric instabilities are evident, associated with the near-boundary convective layer (section 3c). In the center of the domain buoyancy advection by the SI overturning circulation generates a plume of gravitationally unstable fluid which extends towards the top of the boundary layer.

A similar evolution is evident in simulation CI-1, which is configured with the same interior stratification but a steeper slope such that the slope Burger number is $S_{\infty}=1.6$ (table 1). Following the same basic evolution, a downslope Ekman flow develops rapidly at the beginning of the simulation, generating a growing BBL that is associated with reduced stratification and low PV (figure 5). Early in this run $f(f+\partial v / \partial \hat{x})<0$, indicative of centrifugal instability (Haine and Marshall 1998). Later, as the boundary layer adjusts to $q \approx 0$ the flow becomes inertially stable, but the instability continues to gain energy primarily through lateral shear production (section 4), in what can be considered as a mixed SI/CI mode (Wenegrat et al. 2018). 
Notable differences between the two runs include a more rapid shut-down of the cross-slope flow, and a faster growing boundary layer that remains more stratified, in simulation CI-1 compared to SI-1. It will be shown below that these results all follow directly as a consequence of the increased slope-angle, and hence slope Burger number, of CI-1. As in SI-1, overturning cells are evident in the cross-frontal snapshot of CI-1 (figure 6). These instabilities are of a mixed centrifugal-symmetric type (figure 7), growing primarily through energy extracted from the lateral shear of the geostrophic flow (section 4) - enhanced in CI-1 due to the steeper slope angle, which allows the slope-normal shear to project more efficiently on the horizontal — with additional contributions from vertical shear production. The finite amplitude CI thus acts similarly to the SI modes, bringing the boundary layer PV back to zero in what can be considered a forced centrifugal instability, and it will be shown below that indeed the boundary layer evolution is governed by the same essential dynamics, regardless of whether the instabilities are predominantly of the SI or CI type.

\section{Theory of forced SI/CI in the BBL}

To understand the evolution of the boundary layer shown in figures 2 - 6 it is useful to take the mean of the governing equations (1)-(4),

$$
\begin{aligned}
\frac{\partial\langle u\rangle}{\partial t}-f\langle v\rangle & =\langle b\rangle \theta-\frac{\partial\left\langle u^{\prime} w^{\prime}\right\rangle}{\partial z}+v \frac{\partial^{2}\langle u\rangle}{\partial z^{2}}, \\
\frac{\partial\langle v\rangle}{\partial t}+f\langle u\rangle & =-\frac{\partial\left\langle v^{\prime} w^{\prime}\right\rangle}{\partial z}+v \frac{\partial^{2}\langle v\rangle}{\partial z^{2}}, \\
f\langle v\rangle \theta & =-\rho^{-1} \frac{\partial\langle p\rangle}{\partial z}+b-\frac{\partial\left\langle w^{\prime} w^{\prime}\right\rangle}{\partial z}, \\
\frac{\partial\langle b\rangle}{\partial t}+N_{\infty}^{2} \theta\langle u\rangle & =-\frac{\partial\left\langle w^{\prime} b^{\prime}\right\rangle}{\partial z}+\kappa \frac{\partial^{2}\langle b\rangle}{\partial z^{2}},
\end{aligned}
$$

where $\langle\cdot\rangle$ denotes the average over the across-slope $(x)$ direction, and primes indicate departure from the horizontal average. Note that for notational simplicity here, and in the remainder of 
where $J^{z}$ is the slope-normal component of the PV flux,

$$
\mathbf{J}=q \mathbf{u}-\boldsymbol{\omega} \kappa \nabla^{2} b+\nabla b \times v \nabla^{2} \mathbf{u} .
$$


Outside of thin viscous/diffusive layers near the boundary, the PV follows,

$$
\frac{\partial\langle q\rangle}{\partial t}+\frac{\partial\left\langle q^{\prime} w^{\prime}\right\rangle}{\partial z} \simeq 0
$$

Using (11) in (14) then gives,

$$
f \frac{\partial}{\partial t} \frac{\partial\langle b\rangle}{\partial z}+\frac{\partial}{\partial t} \frac{\partial\left\langle\zeta^{\prime} b^{\prime}\right\rangle}{\partial z}-N_{\infty}^{2} \theta \frac{\partial}{\partial t} \frac{\partial\langle v\rangle}{\partial z}+\frac{\partial\left\langle q^{\prime} w^{\prime}\right\rangle}{\partial z} \simeq 0 .
$$

Integrating in the slope-normal direction,

$$
f \frac{\partial}{\partial t}\langle b\rangle+\frac{\partial}{\partial t}\left\langle\zeta^{\prime} b^{\prime}\right\rangle-N_{\infty}^{2} \theta \frac{\partial}{\partial t}\langle v\rangle+\left\langle q^{\prime} w^{\prime}\right\rangle \simeq C(t)
$$

where $C$ is a constant of integration that depends only on time. The perturbation quantities and PV flux go to 0 above the BBL, hence it must be the case that $C(t)=0$.

Using the mean buoyancy equation (10), the PV flux can then be written as ${ }^{1}$,

$$
\left\langle q^{\prime} w^{\prime}\right\rangle \simeq N_{\infty}^{2} \theta\left(\frac{\partial\langle v\rangle}{\partial t}+f\langle u\rangle\right)+f \frac{\partial\left\langle w^{\prime} b^{\prime}\right\rangle}{\partial z}-\frac{\partial\left\langle\zeta^{\prime} b^{\prime}\right\rangle}{\partial t} .
$$

Substituting for the term in parentheses using the mean along-slope momentum balance (8) gives,

$$
\left\langle q^{\prime} w^{\prime}\right\rangle \simeq-N_{\infty}^{2} \theta \frac{\partial\left\langle v^{\prime} w^{\prime}\right\rangle}{\partial z}+f \frac{\partial\left\langle w^{\prime} b^{\prime}\right\rangle}{\partial z}-\frac{\partial\left\langle\zeta^{\prime} b^{\prime}\right\rangle}{\partial t} .
$$

For subinertial motions the last-term on the right-hand side is small relative to the first two terms (following the scaling analysis given in TF10), and hence it can be neglected,

$$
\left\langle q^{\prime} w^{\prime}\right\rangle \simeq-N_{\infty}^{2} \theta \frac{\partial\left\langle v^{\prime} w^{\prime}\right\rangle}{\partial z}+f \frac{\partial\left\langle w^{\prime} b^{\prime}\right\rangle}{\partial z}
$$

For the PV to remain steady in the BBL, the flux must be non-divergent over the BBL, therefore $-N_{\infty}^{2} \theta\left\langle v^{\prime} w^{\prime}\right\rangle+f\left\langle w^{\prime} b^{\prime}\right\rangle$ is at most a linear function of the slope-normal distance (figure 9).

\footnotetext{
${ }^{1}$ Throughout we ignore the molecular diffusive fluxes of buoyancy as they tend to be small relative to other terms. Formally this can be posed (see appendix) as the requirement that $f \kappa\left(1+S_{\infty}^{2}\right) / u^{* 2} \theta \ll 1$, ie the Thorpe transport (Thorpe 1987) is small relative to the Ekman transport, such that advective and resolved turbulent fluxes dominate the diffusive flux. This is generally true, with the exception being the late-time evolution of the large slope Burger number cases, which undergo significant Ekman arrest (section 5 ), such that $u^{*} \rightarrow 0$ and diffusive fluxes can become important. We consider this as somewhat artificial, both due to the enhanced diffusivity used here and the long integration times. Regardless, the cumulative errors due to this approximation remain small in these few cases, hence diffusive terms can be safely ignored.
} 


\section{b. Scaling for the height of the low PV layer}

Once the instabilities have reached finite amplitude in the numerical simulations the boundary layer stratification does not evolve significantly in time, ie. $\partial b / \partial t$ is independent of $z$. Thus, integrating the mean buoyancy equation (10) over a height $H(t)$ from the bottom (again ignoring the small diffusive fluxes of buoyancy),

$$
H \frac{\partial\langle b\rangle}{\partial t} \simeq-\left\langle w^{\prime} b^{\prime}\right\rangle_{z=H}-N_{\infty}^{2} \theta \int_{0}^{H}\langle u\rangle d z .
$$

For the case of a sloping bottom the depth-integrated buoyancy can only be in steady-state when the cross-slope advection exactly balances the buoyancy flux divergence (Thorpe 1987). As the buoyancy perturbation enters the momentum balance, through (7), this implies that there is not necessarily a steady-state solution for any arbitrary Ekman transport, unlike in the surface boundary layer. However, by vertically integrating (7), (8) and (10), it is possible to combine the across and along slope momentum equations to give an approximate equation for the across-slope transport (see appendix, and Brink and Lentz 2010)

$$
\int_{0}^{H}\langle u\rangle d z \simeq-\frac{1}{f\left(1+S_{\infty}^{2}\right)}\left[\frac{\left\langle\tau^{y}\right\rangle}{\rho}+\frac{\theta}{f}\left\langle w^{\prime} b^{\prime}\right\rangle_{z=H}\right],
$$

where $\tau^{y}=\rho v \partial v /\left.\partial z\right|_{z=0}$ is the along-slope bottom stress. The cross-slope transport is therefore given by the BBL Ekman transport, modified to account for the reduction of the Ekman flow by buoyancy forces in the across-slope momentum budget (Brink and Lentz 2010).

Using (21) in (20) gives,

$$
H \frac{\partial\langle b\rangle}{\partial t} \simeq(1+\alpha) E B F_{s},
$$

where we have introduced the slope Ekman Buoyancy Flux,

$$
E B F_{s}=\frac{\left\langle\tau^{y}\right\rangle}{\rho f} \frac{N_{\infty}^{2} \theta}{1+S_{\infty}^{2}},
$$

\footnotetext{
${ }^{2}$ We ignore entrainment fluxes of momentum at $z=H$ for clarity, as they do not contribute significantly in the numerical simulations.
} 
and where $\alpha=-\left\langle w^{\prime} b\right\rangle_{z=H}\left(1+S_{\infty}^{2}\right)^{-1} E B F_{s}^{-1}$ is an entrainment factor accounting for the turbulent buoyancy flux at $z=H$. Practically this term is only important in the simulations dominated by convection, and can otherwise be ignored (section 3c).

The rate of change of buoyancy can be related to the PV flux outside of the near-boundary diffusive layer by using (17) and noting that $\partial\langle v\rangle / \partial t \simeq-f^{-1} \theta \partial\langle b\rangle / \partial t$ (see appendix), such that,

$$
\left\langle q^{\prime} w^{\prime}\right\rangle \simeq-f\left(1+S_{\infty}^{2}\right) \frac{\partial\langle b\rangle}{\partial t} .
$$

Then, defining $H(t)$ as the location where the PV flux vanishes, and integrating (12) vertically gives,

$$
\frac{\partial}{\partial t} \int_{0}^{H(t)}\langle q\rangle d z-\frac{\partial H}{\partial t}\langle q\rangle_{z=H} \simeq-(1+\alpha)\left(1+S_{\infty}^{2}\right) \frac{f E B F_{s}}{H},
$$

where we have used (22) and (24) to write $J_{z=0}^{z} \simeq(1+\alpha)\left(1+S_{\infty}^{2}\right) E B F_{s}$, as the PV flux is assumed constant through the BBL. The rate of change of the integrated boundary layer PV will be small when convective mixing or symmetric/centrifugal instabilities cause $\langle q\rangle \approx 0$. Setting $\langle q\rangle_{z=H}=$ $f N_{\infty}^{2}$, the interior PV, then gives an equation for the rate of change of the thickness of the low PV layer,

$$
H \frac{\partial H}{\partial t} \simeq(1+\alpha)\left(1+S_{\infty}^{2}\right) \frac{E B F_{s}}{N_{\infty}^{2}} .
$$

This can be further simplified as,

$$
H \frac{\partial H}{\partial t}=(1+\alpha) \frac{\left\langle\tau^{y}\right\rangle \theta}{\rho f},
$$

showing how the time evolution of the boundary layer thickness differs from the expectation for upright convection - growing faster by a factor of $1+S_{\infty}^{2}$ (Deardorff et al. 1969) — and depends only weakly on the interior stratification and slope Burger number (through the entrainment fluxes and the bottom stress as discussed in section 5). The accuracy of the boundary layer height predicted by integrating (27) can be seen by comparing the thick black line in the bottom panels of figures 2 and 5 to the depth of the simulated low PV layer. 
c. Scaling for the height of the convective layer

During SI/CI the boundary layer divides into two regions. Near the lower boundary the stratification remains low and turbulent buoyancy fluxes act to increase the eddy kinetic energy — in what is termed the convective layer (TF10) - above which the lies a stratified region where instabilities are active. In some conditions the convective layer can fill the majority of the boundary layer, allowing upright convection to persist even in conditions that otherwise appear conducive to $\mathrm{SI} / \mathrm{CI}$, and it is therefore useful to determine a diagnostic equation for the height of the convective layer, $h(t)$.

In the surface boundary layer the convective layer depth is generally defined as the location where the total vertical buoyancy flux is zero (TF10), however in our simulations we find that this definition does not usefully partition the boundary layer into regions with distinct dynamics. The reason for this can be seen clearly by decomposing the slope-normal buoyancy flux by across-slope wavenumber (figure 9, panel b). Slope-normal buoyancy fluxes with across-slope wavelengths $\lambda_{x}>100 \mathrm{~m}$ are associated with the SI/CI overturning cells, and are positive through a significant portion of the lower boundary layer, whereas fluxes associated with smaller scale turbulent motions $\left(\lambda_{x}<100 \mathrm{~m}\right)$ decay rapidly away from the boundary. The convective layer depth, as commonly defined, is therefore largely determined by the overturning cells of the instability themselves in these simulations, and hence does not discriminate regions of the boundary layer where $\mathrm{SI} / \mathrm{CI}$ is active or not. Detailed exploration of why the instability cells are slightly inclined from isopcynal surfaces, and hence generate buoyancy fluxes is beyond the scope of the present work (see related work by Grisouard 2018). However we note that the regions of positive buoyancy fluxes by SI/CI are partially compensated by negative buoyancy fluxes in the upper boundary layer, such that shear production still dominates the total instability energetics (section 4). 
Given this, we take an alternate definition of the convective layer height as the location at which the small-scale turbulent slope-normal fluxes equal 0 . To do this we decompose the total slope-normal buoyancy fluxes into contributions from SI/CI and turbulent motions, denoted as $\left\langle w^{\prime} b^{\prime}\right\rangle^{I}$ and $\left\langle w^{\prime} b^{\prime}\right\rangle^{T}$ respectively. Then, integrating the mean buoyancy equation (10) to $h(t)$, where $\left\langle w^{\prime} b^{\prime}\right\rangle^{T}=0$ by definition, gives,

$$
\int_{0}^{h(t)} \frac{\partial\langle b\rangle}{\partial t} d z \simeq-N_{\infty}^{2} \theta \int_{0}^{h(t)}\langle u\rangle d z-\left\langle w^{\prime} b^{\prime}\right\rangle_{z=h}^{I}
$$

Recalling that the rate of change of buoyancy is independent of $z$ in the boundary layer, (22) implies,

$$
\frac{h}{H}(1+\alpha) E B F_{s} \simeq-N_{\infty}^{2} \theta \int_{0}^{h(t)}\langle u\rangle d z-\left\langle w^{\prime} b^{\prime}\right\rangle_{z=h}^{I} .
$$

The vertical integral of the cross-slope velocity can be re-written as (see appendix),

$$
\int_{0}^{h(t)}\langle u\rangle d z \simeq \frac{1}{f\left(1+S_{\infty}^{2}\right)}\left[-\left.\left\langle v^{\prime} w^{\prime}\right\rangle\right|_{z=h(t)}-\frac{\left\langle\tau^{y}\right\rangle}{\rho}-\frac{\theta}{f}\left\langle w^{\prime} b^{\prime}\right\rangle_{z=h}^{I}\right],
$$

ie. the cross-slope transport over the layer is proportional to the divergence of the along-slope momentum flux plus a contribution from the buoyancy flux divergence (and where we have ignored small diffusive fluxes of momentum at $z=h$ ). Thus,

$$
\left.\frac{h}{H}(1+\alpha) E B F_{s} \simeq \frac{N_{\infty}^{2} \theta}{f\left(1+S_{\infty}^{2}\right)}\left\langle v^{\prime} w^{\prime}\right\rangle\right|_{z=h}+E B F_{S}-\frac{1}{1+S_{\infty}^{2}}\left\langle w^{\prime} b^{\prime}\right\rangle_{z=h}^{I}
$$

Solving this equation directly for $h$ using numerical estimates of $\left\langle v^{\prime} w^{\prime}\right\rangle_{z=h}$ and $\left\langle w^{\prime} b^{\prime}\right\rangle_{z=h}^{I}$ (defined using a cutoff wavelength of $\lambda_{x}=100 \mathrm{~m}$ and excluding cases where $E B F_{s} / \kappa N^{2}<\left(1+S_{\infty}^{2}\right)$ for consistency with the assumptions used in the derivation) shows excellent agreement with the true convective layer depth across all simulations $\left(r^{2}=0.98\right)$.

To close this equation for diagnostic purposes it is necessary to estimate the eddy momentum and buoyancy flux terms. To do this we assume that the slope-normal buoyancy flux term generated by $\mathrm{SI} / \mathrm{CI}$ is proportional to the $E B F_{s}$, the along-front turbulent velocity scale goes like the change in 
geostrophic velocity over the convective layer $v^{\prime} \sim h \partial v_{g} / \partial z$, and the vertical velocity scales with the convective velocity $w^{\prime} \sim\left(E B F_{s} h\right)^{1 / 3}$ (as in TF10). The $q \approx 0$ condition provides a constraint on the perturbation buoyancy gradient in the boundary layer (assuming linear variation of buoyancy through the boundary layer, as in figure 8, and Allen and Newberger 1998),

$$
\frac{\partial\langle b\rangle}{\partial z} \simeq-\frac{N_{\infty}^{2}}{1+S_{\infty}^{2}} .
$$

Noting that the geometry of the problem gives $\partial\langle b\rangle / \partial \hat{x}=-\theta \partial\langle b\rangle / \partial z$, the thermal wind shear can then be written as $\partial\left\langle v_{g}\right\rangle / \partial \hat{z} \simeq N_{\infty}^{2} \theta / f\left(1+S_{\infty}^{2}\right)$. Using these relationships and scalings, the equation governing the convective layer depth can be written,

$$
\left(\frac{h}{H}\right)^{4}-C^{3}\left(\frac{u^{* 2}}{\Delta v_{g}^{2}} \cos \gamma\right)^{2}\left[1-(1+\alpha) \frac{h}{H}\right]^{3}=0,
$$

where $C$ is a constant with best estimate determined from fitting the numerical simulations of $C=$ 8.3 (figure 10), $\gamma$ is the angle of the bottom stress relative to the along-slope direction, and $\Delta v_{g}=$ $H N^{2} \theta / f\left(1+S_{\infty}^{2}\right)$ is the change in geostrophic velocity over the boundary layer height. Aside from slight differences in the best-fit coefficient, this equation is the same as for the convective depth in the surface boundary layer in the case of downfront winds and no surface buoyancy loss (TF10; Thomas et al. 2013). Alternate definitions of the cutoff wavelength, $\lambda_{x}$, were tested and found to lead to only minor quantitative changes in the best-fit coefficient.

The convective layer height is therefore controlled by the term $u^{*} / \Delta v_{g}$, the ratio of the friction velocity to the change in geostrophic velocity over the BBL. An alternate expression of this utilizes the slope Monin-Obukhov length (Ruan et al. 2019),

$$
L_{s}=\frac{u^{* 3}}{\mathscr{K} E B F_{s}},
$$

where $\mathscr{K}=0.4$ is the von Kármán constant, such that $u^{*} / \Delta v_{g}=\mathscr{K} L_{S} / H$. Thus, when $L_{s} / H \ll 1$ the first term in (33) dominates, and the convective layer depth goes to 0 . When $L_{s} / H \gg 1$ only 
the second term in (33) contributes, and the convective layer fills the boundary layer outside an entrainment layer near the boundary layer top, such that $h \approx H /(1+\alpha)$. An example of this latter case is shown in figure 11 for simulation CONV-1, where $L s / H \gg 1$, and SI/CI are absent and the boundary layer is instead characterized by gravitational instability (figure 12).

Conditions of $f q<0$ are therefore not independently sufficient for SI/CI in the BBL, and it is additionally necessary that $h / H \ll 1$. This final criteria is satisfied when the change in geostrophic velocity over the boundary layer height is much larger than the friction velocity $\left(L_{S} / H \ll 1\right)$, similar to the criteria for wind-forced SI in the surface boundary layer (Thomas et al. 2013). However, unlike the surface boundary layer case, in the BBL these two quantities are not independent, as increasing $\Delta v_{g}$ acts to decrease the bottom stress, discussed further in section 5.

\section{Energetics}

In the slope-coordinate system the eddy kinetic energy (EKE) budget is,

$$
\frac{\partial\langle k\rangle}{\partial t}=\underbrace{\left\langle w^{\prime} b^{\prime}\right\rangle+\left\langle u^{\prime} b^{\prime}\right\rangle \theta}_{V B P} \underbrace{-\left\langle v^{\prime} w^{\prime}\right\rangle \frac{\partial\langle v\rangle}{\partial z}-\left\langle u^{\prime} w^{\prime}\right\rangle \frac{\partial\langle u\rangle}{\partial z}}_{S P} \underbrace{-\frac{\partial}{\partial z}\left(\left\langle w^{\prime} k^{\prime}\right\rangle+\left\langle w^{\prime} p^{\prime}\right\rangle-v \frac{\partial\langle k\rangle}{\partial z}\right)}_{\text {TRANSPORT }} \underbrace{-\varepsilon}_{\text {DISS }},
$$

where $k=\left(u^{\prime 2}+v^{\prime 2}+w^{\prime 2}\right) / 2$ is the EKE, $\varepsilon=v\left\langle s_{i, j}^{\prime} s_{i, j}^{\prime}\right\rangle$ is the dissipation rate, and $s_{i, j}^{\prime}=$ $\left(\partial u_{i}^{\prime} / \partial x_{j}+\partial u_{j}^{\prime} / \partial x_{i}\right) / 2$ is the strain tensor. Terms in the budget are, from left to right, the vertical buoyancy production (VBP, which involves both slope-normal and across-slope fluxes), shear production (SP), transport of EKE (TRANSPORT), and dissipation of eddy kinetic energy (DISS).

To further simplify the budget, the shear production term can be decomposed into geostrophic and ageostrophic components. The governing equations for the mean shear (assuming sub-inertial 


\footnotetext{
${ }^{3}$ Note that when considering the horizontal momentum equations the rate of change of buoyancy still influences the along-slope momentum balance, and hence it is necessary to retain the rate of change terms, as discussed in the appendix. However here, where we consider the equations governing the mean slope-normal shear, the assumptions that momentum evolves on subinertial timescales and that $\partial^{2}\langle b\rangle / \partial z \partial t \approx 0$, together allow the rate of change terms to be neglected.
} 
terms of standard vertical and lateral shear production terms. Using the fact that the fastest growing mode is aligned along isopycnals (Thomas et al. 2013), which have slope $\partial z /\left.\partial x\right|_{\rho} \approx-\theta S_{\infty}^{-2}$ when $q \approx 0$ (Allen and Newberger 1998), the ratio of the lateral geostrophic shear production (LGSP) to the vertical geostrophic shear production (VGSP) will be,

$$
\frac{L G S P}{V G S P} \sim\left(\left.\frac{\partial z}{\partial x}\right|_{\rho}\right)^{-1} \theta \simeq S_{\infty}^{2}
$$

The same result can also be derived directly from the definition of the PV, which, assuming that the flow is in approximate geostrophic balance, can be written,

$$
q \simeq f N^{2}\left(1+R o_{b}-\frac{1}{R i_{b}}\right)
$$

where $R o_{b}=f^{-1} \partial v_{g} / \partial \hat{x}$ and $R i_{b}=N^{2}\left(\partial v_{g} / \partial \hat{z}\right)^{-2}$ are the balanced Rossby and Richardson number, respectively. The product of these terms thus determines whether the PV is vortically low (associated with CI), or baroclinically low (associated with SI, Thomas 2008). Using (32), and the relationship $\partial v_{g} / \partial \hat{x}=-\theta \partial v_{g} / \partial \hat{z}$

$$
R o_{b} R i_{b}=S_{\infty}^{2}
$$

Thus, both the energetics and PV indicate that centrifugal-type instabilities are expected to dominate when the interior slope Burger number exceeds 1 (for instance run CI-1, figure 13), and symmetric-type instabilities will dominate when $S_{\infty}<1$ (e.g. run SI-1).

Importantly, while the SI/CI modes grow via $G S P_{s}$, much of the total energy extracted from the geostrophic flow via shear production is balanced directly by dissipation. An example of this is shown in figure 14 for simulation SI-1, where the rate of change of EKE is a small residual between the near compensation of shear production and dissipation. It is therefore of interest to constrain the magnitude and vertical structure of the combined EKE production terms, as these set the dissipation rate in the boundary layer. In the surface boundary layer these follow directly from the PV flux equation (TF10; Thomas and Taylor 2010), however, in the BBL case a few additional 
steps are necessary. First, consider the eddy potential energy equation, ignoring vertical transport terms for simplicity,

$$
\frac{\partial}{\partial t}\left(\frac{\left\langle b^{\prime 2}\right\rangle}{2 N_{\infty}^{2}}\right)=\underbrace{-\left\langle w^{\prime} b^{\prime}\right\rangle-\left\langle u^{\prime} b^{\prime}\right\rangle \theta}_{-V B P}-\underbrace{\frac{\left\langle w^{\prime} b^{\prime}\right\rangle}{N_{\infty}^{2}} \frac{\partial\langle b\rangle}{\partial z}}_{M P E-E P E}-\underbrace{\frac{\kappa}{N_{\infty}^{2}}\left\langle\frac{\partial b^{\prime}}{\partial z} \frac{\partial b^{\prime}}{\partial z}\right\rangle}_{D I S S_{b}} .
$$

The first term on the right-hand side is the negative of the vertical buoyancy production term, representing the loss of eddy potential energy to eddy kinetic energy, the second term is the conversion between mean and eddy potential energy (MPE-EPE), and the final term gives the rate of irreversible mixing of buoyancy $\left(\right.$ DISS $\left._{\mathrm{b}}\right)$.

The numerical simulations show that, when in the forced-SI/CI regime, both the rate of change of EPE and DISS $\mathrm{b}_{\mathrm{b}}$ are small. Hence the EPE budget can be approximated as,

$$
0 \simeq-\left\langle w^{\prime} b^{\prime}\right\rangle-\left\langle u^{\prime} b^{\prime}\right\rangle \theta-\frac{\left\langle w^{\prime} b^{\prime}\right\rangle}{N_{\infty}^{2}} \frac{\partial\langle b\rangle}{\partial z} .
$$

Using (32), this gives,

$$
\frac{\left\langle w^{\prime} b^{\prime}\right\rangle}{1+S_{\infty}^{2}} \simeq\left\langle w^{\prime} b^{\prime}\right\rangle+\left\langle u^{\prime} b^{\prime}\right\rangle \theta .
$$

Physically this states that in the limit where both the rate of change and dissipation of EPE are small, conversions between eddy potential and kinetic energy are balanced by conversions between mean and eddy potential energy. Finally, using (32) the $G S P_{s}$ can be expressed as $G S P_{s} \approx-\left\langle v^{\prime} w^{\prime}\right\rangle N_{\infty}^{2} \theta / f\left(1+S_{\infty}^{2}\right)$, allowing the EKE budget to be approximated as,

$$
\frac{\partial\langle k\rangle}{\partial t} \simeq \frac{1}{1+S_{\infty}^{2}}\left[\left\langle w^{\prime} b^{\prime}\right\rangle-\left\langle v^{\prime} w^{\prime}\right\rangle \frac{N_{\infty}^{2} \theta}{f}\right]-\varepsilon .
$$

From the PV flux equation (19) the first term on the right-hand side of (46) is a linear function of $z$, with maximum value given by $E B F_{S}$ (figure 9). In the case that the rate of change of EKE is small, this implies that the dissipation must also be a linear function of $z$, with magnitude set by 
the slope Ekman buoyancy flux (figure 15),

$$
\varepsilon_{S I} \approx \begin{cases}E B F_{S}\left(1-\frac{z}{H}\right), & \text { if } z \leq H \\ 0, & \text { otherwise. }\end{cases}
$$

The vertically integrated dissipation from SI/CI in the BBL will therefore go as $(H / 2) E B F_{s}$. A comparison of the depth-integrated production terms, first term in (46), to the parameterized depthintegrated dissipation is shown in figure 15c. The agreement is excellent across all simulations, although the dissipation is overestimated by approximately $10 \%$, likely due to production terms that go to zero near the lower boundary more smoothly than predicted by the piecewise approximation given by (47). A similar result for SI in the surface boundary layer has proven useful in explaining observations of enhanced turbulent dissipation at symmetrically unstable fronts (D'Asaro et al. 2011; Thomas et al. 2016), and for the development of parameterizations of unresolved SI turbulence (Bachman et al. 2017).

\section{Symmetric/centrifugal instability and Ekman buoyancy arrest}

Above it is shown that during the Ekman adjustment process of the boundary layer the flow quickly becomes unstable to SI/CI, which grow to finite amplitude and begin to modify the dynamical evolution of the boundary layer. It is therefore of interest to consider how the presence of these instabilities modifies the classic picture of Ekman buoyancy arrest (MacCready and Rhines 1991, 1993; Brink and Lentz 2010). The most obvious modification to the Ekman arrest process by SI/CI is through the enhanced stratification of the boundary layer necessary to bring the PV to the point of marginal stability $(q \approx 0)$. As noted by Allen and Newberger $(1998)$ this modifies the depth of the BBL necessary to achieve full Ekman arrest,

$$
H_{a}=\frac{V_{\infty} f\left(1+S_{\infty}^{2}\right)}{N_{\infty}^{2} \theta},
$$




\footnotetext{
${ }^{4}$ The relaminarization height (Ruan et al. 2019) of the boundary layer, which marks the point at which turbulence in the boundary layer is suppressed by viscous effects, will similarly be increased by a factor of $1+S_{\infty}^{2}$ by SI/CI. This can be seen by replacing the approximate stress relation in Ruan et al. (2019, their equation 13) with $\tau^{y} / \rho \approx C_{d}\left[V_{\infty}-H N_{\infty}^{2} \theta / f\left(1+S_{\infty}^{2}\right)\right]^{2}$ to reflect the reduced geostrophic shear.
} 
timescale can also be compared to that for classic Ekman arrest (ie. $R i_{c}=0$ ) where,

$$
T_{E}=\frac{V_{\infty}^{2}\left(1+S_{\infty}^{2}\right)}{2 N_{\infty} S_{\infty}^{3} u_{o}^{* 2}} .
$$

SI/CI thus extends the arrest process by a factor of $1+S_{\infty}^{2}$ via restratification of the boundary layer, which reduces the strength of the thermal wind shear.

A detailed analysis of the energetics of Ekman adjustment of the BBL in the presence of SI/CI will be the subject of a future manuscript, however it is worth briefly noting the effect that these processes may have on the energetics of the general ocean circulation, where bottom drag over topography is believed to be a key sink of kinetic energy from the balanced flow field (Ferrari and Wunsch 2009; Sen et al. 2008; Arbic et al. 2009). The combined bottom drag on the geostrophic flow and vertically integrated dissipation due to SI can be conceptualized as an effective drag (cf. Thomas and Taylor 2010),

$$
D R A G_{E F F}=\left.\tau^{y} v_{g}\right|_{z=0}+\int_{0}^{\infty} \varepsilon_{S I} \mathrm{dz},
$$

which, using (47), and the definition of the change in geostrophic velocity across the boundary layer, $\Delta v_{g}=H N_{\infty}^{2} \theta / f\left(1+S_{\infty}^{2}\right)$, can be written as,

$$
D R A G_{E F F}=\tau^{y}\left(\left.v_{g}\right|_{z=0}+\frac{1}{2} \Delta v_{g}\right) .
$$

Considering the development of thermal wind shear during the Ekman arrest process, which reduces the bottom geostrophic velocity from the interior values such that $\left.v_{g}\right|_{z=0}=V_{\infty}-\Delta v_{g}$, the effective drag can also be written as, $D R A G_{E F F}=\tau^{y}\left(V_{\infty}-\Delta v_{g} / 2\right)$. Thus, while the Ekman arrest process reduces the drag on the geostrophic flow through the development of thermal wind shear, the presence of SI/CI offsets half of this reduction directly through enhanced dissipation of kinetic energy extracted from the geostrophic flow either directly through $G S P_{S}$ or indirectly through the release of available potential energy (which in the Ekman arrest process is ultimately sourced from the mean kinetic energy, Umlauf et al. 2015). 


\section{Summary and Discussion}

Recently there has been a renewed interest in the dynamics of the BBL, motivated in part by the possibility that recent advances in understanding submesoscale processes at the ocean's surface might also provide insight into the physical processes at the bottom (McWilliams 2016; Wenegrat et al. 2018). In this manuscript we focused on the case of an interior flow along isobaths of a sloping lower boundary which generates a downslope Ekman transport, as a BBL counterpart to the well-studied case of downfront surface wind stress. We show that there exists a state of forced centrifugal and symmetric instability in the BBL, which behaves much like the state of forced symmetric instability in the surface boundary layer (TF10; Thomas and Taylor 2010). Importantly, the fact that the $\mathrm{BBL}$ evolves to reach the state of marginal stability to SI/CI (ie. $q \approx 0$ ) provides a strong constraint on the evolution, with major consequences including:

1. The slope Ekman buoyancy flux, $E B F_{S}(23)$, controls both the rate of change of buoyancy in the boundary layer (22), and the slope-normal flux of PV (19). This allows the governing equation for the height of the low PV layer to be expressed as a simple ordinary-differential equation involving the bottom stress, slope angle, and Coriolis frequency (27).

2. SI/CI restratifies the BBL, such that the approximate stratification of the boundary layer goes as $N_{\infty}^{2} S_{\infty}^{2} /\left(1+S_{\infty}^{2}\right)$ (Allen and Newberger 1998). Thus, the BBL may retain significant stratification, particularly in regimes with large interior slope Burger numbers. This finding should be considered when interpreting observations, as our results suggest significant turbulent dissipation via SI/CI is possible even in stratified regions that would not necessarily be easily identifiable as a boundary layer in terms of the buoyancy profile alone. For example in observations of SI/CI unstable conditions in the deep Orkney Passage (Naveira Garabato et al. 
2019) interior slope Burger numbers of $S_{\infty} \approx 1.4$ suggest that SI/CI may be active in regions where the stratification is as large as $2 / 3$ of the interior values.

3. Downslope Ekman transport always tends to generate conditions unstable to SI/CI through the destruction of boundary layer PV. However, it is also necessary to consider the ratio of the slope Monin-Obukhov length, $L_{s}$ (34), to the boundary layer depth when evaluating whether SI/CI will be present - specifically when $L_{S} / H$ is large the boundary layer remains unstratified and SI/CI is absent. We note however that in the case that $L_{S} / H$ is large because $S_{\infty}$ is small, baroclinic instabilities are likely to emerge rapidly (though not present in the 2D simulations used here) (Brink and Cherian 2013; Wenegrat et al. 2018).

4. The primary energy source for SI/CI in the BBL is the slope Geostrophic Shear Production, $G S P_{S}$ (39), whereby slope-normal eddy fluxes extract energy from the background geostrophic shear. The energy source for the BBL instabilities can therefore involve mixed SI/CI modes with energy extracted from the geostrophic flow through both lateral and vertical shear production terms (40). The slope Burger number provides an indicator of whether the instability will be of the centrifugal $\left(S_{\infty}^{2}>1\right)$ or symmetric $\left(S_{\infty}^{2}<1\right)$ type, (40) and (41).

5. The dissipation rate in the boundary layer due to SI/CI scales with the $E B F_{s}$, and decreases linearly through the boundary layer height (47), hence the integrated SI/CI dissipation goes as $(H / 2) E B F_{s}$. In the surface boundary layer similar results (eg. Thomas and Taylor 2010) have been used as the basis for parameterization for models that do not directly resolve SI (Bachman et al. 2017), and our results suggest a similar parameterization is possible for the BBL.

6. SI/CI extends the Ekman arrest time by a factor of $\left(1+S_{\infty}^{2}\right)$, and increases the arrested Ekman height by the same factor, but does not stop the buoyancy arrest process. The total loss of 
energy from the balanced flow through bottom drag and SI/CI during Ekman arrest can be conceptualized as an effective bottom drag (52), which shows that energy extraction from the geostrophic shear by SI/CI offsets exactly half of the reduction in bottom stress due to the development of thermal wind shear in the boundary layer. SI/CI also increases the timeintegrated bottom drag by slowing the Ekman arrest process, ie. slowing the decay of the bottom stress.

Beyond instabilities of the BBL itself, a variety of recent work has also noted that the formation of topographic wakes, characterized by the shedding of BBL fluid with $f q<0$, appears to be a common feature in realistic submesoscale-resolving simulations (Molemaker et al. 2015; Dewar et al. 2015; Gula et al. 2016; Srinivasan et al. 2019). These topographic wakes appear to be particularly susceptible to $\mathrm{CI}$, which generate dissipation rates that may be sufficiently large to affect the energetics of regional or even global ocean circulation (Gula et al. 2016). The development of these wake instabilities will be sensitive to the upstream BBL evolution, and hence they may also be influenced by SI/CI in the BBL. For instance, when BBL instabilities are able to bring the boundary layer to the state of $q \approx 0$ before boundary layer separation, the subsequent topographic wake can be stabilized to further instabilities. A manuscript detailing how the instabilities and energetics of topographic wakes depends on the upstream BBL evolution is currently in preparation.

One additional aspect of how BBL instabilities can modulate flow-topography interaction which was not a specific focus of the work presented here — is by affecting the irreversible mixing of buoyancy along topography. This topic has broad implications for the large-scale ocean circulation, and, for example, recent observational and numerical modeling work has suggested that submesoscale instabilities along topography may play an important role in the deep-overturning circulation (Ruan et al. 2017; Wenegrat et al. 2018; Callies 2018; Naveira Garabato et al. 2019). 
The distinction between SI and CI modes, which we argued above was somewhat artificial in regards to the kinetic energy budget, may be of more significance when considering the mixing of buoyancy. Specifically, simulations of interior CI suggest very high mixing efficiencies (Jiao and Dewar 2015), in contrast to the SI modes which are aligned primarily along isopcynals and hence tend to have very low mixing efficiencies. Further investigation of SI/CI in the BBL and topographic wakes will help clarify the role of submesoscale instabilities in watermass transformation along topography.

Acknowledgments. J.O.W. thanks Bertrand Delorme, John Taylor, and Andrew Thompson for stimulating discussions during the preparation of this manuscript. Funding for this work was provided by NSF grant OCE-1756118. All numerical simulation output used in this manuscript was created using the open source software code Dedalus, which is available for download from http://dedalus-project.org/.

In this appendix we derive the approximate cross-slope transport equation, (21). This follows closely from Brink and Lentz (2010), however we integrate over a finite depth, and retain entrainment fluxes of buoyancy. First, taking the time-derivative of (7), and combining with (8), gives a single expression that combines the horizontal momentum equations,

$$
\frac{\partial^{2}\langle u\rangle}{\partial t^{2}}+f^{2}\langle u\rangle=f \frac{\partial F^{y}}{\partial z}+\frac{\partial^{2} F^{x}}{\partial z \partial t}+\frac{\partial\langle b\rangle}{\partial t} \theta
$$

where $F^{x}=-\left\langle u^{\prime} w^{\prime}\right\rangle+v \partial\langle u\rangle / \partial z$ is the combined turbulent and diffusive slope-normal flux of across-slope momentum, and $F^{y}$ is defined similarly for the along-slope momentum.

Variables in this equation can be scaled as $u \sim U, t \sim T, z \sim H, F^{y} \sim \tau^{y} / \rho_{o}, F^{x} \sim \tau^{x} / \rho_{o}$, and $b \sim T U N_{\infty}^{2} \theta$. This scaling for the buoyancy is a consequence of the assumption that in the regimes 
of interest here the across-slope advection of buoyancy is leading-order in the mean buoyancy equation (10). Using these scalings, the ratio of the first term on the left hand side to the Coriolis acceleration is

$$
\frac{U}{U T^{2} f^{2}} \sim O\left(T^{2} f^{2}\right)^{-1}
$$

The ratio of the second term on the right-hand side to the first term on the right-hand side is,

$$
\frac{\tau^{x} H}{\tau^{y} f T H} \leq O(T f)^{-1}
$$

where we have assumed that $\tau_{x} \leq \tau_{y}$ as the interior velocity is aligned in the y-direction. Thus, both the first term on the left-hand side, and the second-term on the right hand side can be neglected when considering subinertial motions where $T f \gg 1$. In contrast, the last term on the right-hand side, involving the perturbation buoyancy, scales relative to the Coriolis acceleration as,

$$
\frac{T U N^{2} \theta^{2}}{f^{2} T U} \sim S_{\infty}^{2},
$$

which is not necessarily small (table 1). We thus neglect time-dependence of the across-slope momentum and stress, while retaining the influence of buoyancy on the across-slope momentum equation, as in Brink and Lentz (2010), such that

$$
f^{2}\langle u\rangle \simeq f \frac{\partial F^{y}}{\partial z}+\frac{\partial\langle b\rangle}{\partial t} \theta .
$$

Now integrate over a layer of thickness $z^{\prime}$, using the mean buoyancy equation (10) to replace the rate of change of buoyancy,

$$
\left.f^{2}\left(1+S_{\infty}^{2}\right) \int_{0}^{z^{\prime}}\langle u\rangle \mathrm{dz} \simeq f F^{y}\right|_{z=z^{\prime}}-f \frac{\left\langle\tau^{y}\right\rangle}{\rho}-\theta\left\langle w^{\prime} b^{\prime}\right\rangle_{z=z^{\prime}}+\left.\theta \kappa \frac{\partial\langle b\rangle}{\partial z}\right|_{z=z^{\prime}}+\theta \kappa N_{\infty}^{2} .
$$

564 relative to the bottom stress as

$$
\frac{\kappa N_{\infty}^{2} \theta \rho}{f \tau^{y}}=\frac{\kappa \rho f}{\theta \tau^{y}} S_{\infty}^{2}<\frac{\kappa \rho f}{\theta \tau^{y}}\left(1+S_{\infty}^{2}\right)
$$


ie. the ratio of the Thorpe transport (Thorpe 1987), $\kappa / \theta$, to the standard Ekman transport, $\tau^{y} / \rho f$, times the squared interior slope Burger number. The final inequality is included to indicate the ratio of the boundary diffusive flux of buoyancy to the advective flux that appears in the buoyancy equation (10), which provides a stronger constraint. These ratios are both generally very small, hence we neglect the diffusive flux of buoyancy. However we note that if desired it is straightforward to incorporate viscous/diffusive fluxes into the theory developed here. Similar arguments also allow for ignoring the diffusive flux of momentum at $z^{\prime}$. In contrast the resolved turbulent buoyancy and momentum fluxes scale with the $E B F_{s}$ and bottom stress, and are therefore not necessarily small, depending on where in the boundary layer $z^{\prime}$ is taken to be.

Thus, an approximate form for the depth integrated cross-slope transport equation is,

$$
f^{2}\left(1+S_{\infty}^{2}\right) \int_{0}^{z^{\prime}} u \mathrm{dz} \simeq-f\left\langle v^{\prime} w^{\prime}\right\rangle_{z=z^{\prime}}-f \frac{\left\langle\tau^{y}\right\rangle}{\rho}-\theta\left\langle w^{\prime} b^{\prime}\right\rangle_{z=z^{\prime}}
$$

\section{References}

Allen, J. S., and P. A. Newberger, 1993: Downwelling Circulation on the Oregon Continental Shelf. Part I: Response to Idealized Forcing. Journal of Physical Oceanography, 26, 20112035, doi:10.1175/1520-0485(1996)026〈2011:DCOTOC >2.0.CO;2.

Allen, J. S., and P. A. Newberger, 1998: On Symmetric Instabilities in Oceanic Bottom Boundary Layers. Journal of Physical Oceanography, 28 (6), 1131-1151, doi:10.1175/1520-0485(1998) 028〈1131:OSIIOB $\rangle$ 2.0.CO;2, URL http://journals.ametsoc.org/doi/abs/10.1175/1520-0485\% $281998 \% 29028 \% 3 \mathrm{C} 1131 \% 3 \mathrm{AOSIIOB} \% 3 \mathrm{E} 2.0 . \mathrm{CO} \% 3 \mathrm{~B} 2$.

Arbic, B. K., and Coauthors, 2009: Estimates of bottom flows and bottom boundary layer dissipation of the oceanic general circulation from global high-resolution models. Journal of Geo- 
physical Research, 114 (C2), C02 024, doi:10.1029/2008JC005072, URL http://doi.wiley.com/ 10.1029/2008JC005072.

Bachman, S., B. Fox-Kemper, J. Taylor, and L. Thomas, 2017: Parameterization of Frontal Symmetric Instabilities. I: Theory for Resolved Fronts. Ocean Modelling, 109, 72-95, doi:10.1016/ j.ocemod.2016.12.003, URL http://linkinghub.elsevier.com/retrieve/pii/S1463500316301482.

Benthuysen, J., and L. N. Thomas, 2012: Friction and Diapycnal Mixing at a Slope: Boundary Control of Potential Vorticity. Journal of Physical Oceanography, 42 (9), 1509-1523, doi:10. 1175/JPO-D-11-0130.1, URL http://journals.ametsoc.org/doi/abs/10.1175/JPO-D-11-0130.1.

Brink, K. H., and D. A. Cherian, 2013: Instability of an idealized tidal mixing front: Symmetric instabilities and frictional effects. Journal of Marine Research, 71 (6), 425-450, doi: 10.1357/002224013812587582, URL http://openurl.ingenta.com/content/xref?genre=article\& issn $=0022-2402 \&$ volume $=71 \&$ issue $=6 \&$ spage $=425$.

Brink, K. H., and S. J. Lentz, 2010: Buoyancy Arrest and Bottom Ekman Transport. Part I: Steady Flow. Journal of Physical Oceanography, 40 (4), 621-635, doi:10.1175/2009JPO4266.1, URL http://journals.ametsoc.org/doi/abs/10.1175/2009JPO4266.1.

Burns, K. J., G. M. Vasil, J. S. Oishi, D. Lecoanet, and B. Brown, 2016: Dedalus: Flexible framework for spectrally solving differential equations. Astrophysics Source Code Library, ascl:1603.015, URL http://adsabs.harvard.edu/abs/2016ascl.soft03015B.

Burns, K. J., G. M. Vasil, J. S. Oishi, D. Lecoanet, and B. P. Brown, 2019: Dedalus: A Flexible Framework for Numerical Simulations with Spectral Methods. arXiv:1905.10388 [astro-ph, physics:physics], URL http://arxiv.org/abs/1905.10388, arXiv: 1905.10388. 
Callies, J., 2018: Restratification of Abyssal Mixing Layers by Submesoscale Baroclinic Eddies. Journal of Physical Oceanography, 48 (9), 1995-2010, doi:10.1175/JPO-D-18-0082.1, URL http://journals.ametsoc.org/doi/10.1175/JPO-D-18-0082.1.

D’Asaro, E., C. Lee, L. Rainville, R. Harcourt, and L. Thomas, 2011: Enhanced Turbulence and Energy Dissipation at Ocean Fronts. Science, 332 (6027), 318-322, doi:10.1126/science. 1201515, URL http://www.sciencemag.org/cgi/doi/10.1126/science.1201515.

Deardorff, J. W., G. E. Willis, and D. K. Lilly, 1969: Laboratory investigation of non-steady penetrative convection. Journal of Fluid Mechanics, 35 (1), 7-31, doi:10.1017/S0022112069000942, URL https://www.cambridge.org/core/product/identifier/ S0022112069000942/type/journal_article.

Dewar, W. K., J. C. McWilliams, and M. J. Molemaker, 2015: Centrifugal Instability and Mixing in the California Undercurrent. Journal of Physical Oceanography, 45 (5), 1224-1241, doi: 10.1175/JPO-D-13-0269.1, URL http://journals.ametsoc.org/doi/10.1175/JPO-D-13-0269.1.

Ferrari, R., and C. Wunsch, 2009: Ocean Circulation Kinetic Energy: Reservoirs, Sources, and Sinks. Annual Review of Fluid Mechanics, 41 (1), 253-282, doi:10.1146/annurev.fluid. 40.111406.102139, URL http://www.annualreviews.org/doi/10.1146/annurev.fluid.40.111406. 102139.

Garrett, C., P. MacCready, and P. Rhines, 1993: Boundary Mixing and Arrested Ekman Layers: Rotating Stratified Flow Near a Sloping Boundary. Annual Review of Fluid Mechanics, 25 (1), 291-323, doi:10.1146/annurev.fl.25.010193.001451, URL http://www.annualreviews. org/doi/10.1146/annurev.fl.25.010193.001451. 
Grisouard, N., 2018: Extraction of Potential Energy from Geostrophic Fronts by Inertial-Symmetric Instabilities. Journal of Physical Oceanography, 48 (5), 1033-1051, doi: 10.1175/JPO-D-17-0160.1, URL http://journals.ametsoc.org/doi/10.1175/JPO-D-17-0160.1.

Gula, J., M. J. Molemaker, and J. C. McWilliams, 2016: Topographic generation of submesoscale centrifugal instability and energy dissipation. Nature Communications, 7, 12 811, doi:10.1038/ ncomms12811, URL http://www.nature.com/doifinder/10.1038/ncomms12811.

Haine, T. W. N., and J. Marshall, 1998: Gravitational, Symmetric, and Baroclinic Instability of the Ocean Mixed Layer. Journal of Physical Oceanography, 28 (4), 634-658, doi: 10.1175/1520-0485(1998)028〈0634:GSABIO $\rangle 2.0 . C O ; 2$, URL http://journals.ametsoc.org/doi/ abs/10.1175/1520-0485\%281998\%29028\%3C0634\%3AGSABIO\%3E2.0.CO\%3B2.

Jiao, Y., and W. K. Dewar, 2015: The Energetics of Centrifugal Instability. Journal of Physical Oceanography, 45 (6), 1554-1573, doi:10.1175/JPO-D-14-0064.1, URL http://journals. ametsoc.org/doi/10.1175/JPO-D-14-0064.1.

MacCready, P., and P. B. Rhines, 1991: Buoyant inhibition of Ekman transport on a slope and its effect on stratified spin-up. Journal of Fluid Mechanics, 223 (-1), 631, doi:10.1017/ S0022112091001581, URL http://www.journals.cambridge.org/abstract_S0022112091001581.

MacCready, P., and P. B. Rhines, 1993: Slippery Bottom Boundary Layers on a Slope. Journal of Physical Oceanography, 23 (1), 5-22, doi:10.1175/1520-0485(1993)023<0005: SBBLOA 2.0.CO;2, URL http://journals.ametsoc.org/doi/abs/10.1175/1520-0485\%281993\% 29023\%3C0005\%3ASBBLOA\%3E2.0.CO\%3B2.

McWilliams, J. C., 2016: Submesoscale currents in the ocean. Proceedings of the Royal Society A: Mathematical, Physical and Engineering Science, 472 (2189), 20160 117, doi:10.1098/rspa. 
2016.0117, URL http://rspa.royalsocietypublishing.org/lookup/doi/10.1098/rspa.2016.0117.

Molemaker, M. J., J. C. McWilliams, and W. K. Dewar, 2015: Submesoscale Instability and Generation of Mesoscale Anticyclones near a Separation of the California Undercurrent. Journal of Physical Oceanography, 45 (3), 613-629, doi:10.1175/JPO-D-13-0225.1, URL http: //journals.ametsoc.org/doi/abs/10.1175/JPO-D-13-0225.1.

Naveira Garabato, A. C., and Coauthors, 2019: Rapid mixing and exchange of deep-ocean waters in an abyssal boundary current. Proceedings of the National Academy of Sciences, 116 (27), 13 233-13 238, doi:10.1073/pnas.1904087116, URL http://www.pnas.org/lookup/doi/10.1073/ pnas. 1904087116.

Ruan, X., A. F. Thompson, M. M. Flexas, and J. Sprintall, 2017: Contribution of topographically generated submesoscale turbulence to Southern Ocean overturning. Nature Geoscience, 10 (11), 840-845, doi:10.1038/ngeo3053, URL http://www.nature.com/doifinder/10.1038/ngeo3053.

Ruan, X., A. F. Thompson, and J. R. Taylor, 2019: The Evolution and Arrest of a Turbulent Stratified Oceanic Bottom Boundary Layer over a Slope: Downslope Regime. Journal of Physical Oceanography, 49 (2), 469-487, doi:10.1175/JPO-D-18-0079.1, URL http://journals.ametsoc. org/doi/10.1175/JPO-D-18-0079.1.

Sen, A., R. B. Scott, and B. K. Arbic, 2008: Global energy dissipation rate of deep-ocean lowfrequency flows by quadratic bottom boundary layer drag: Computations from current-meter data. Geophysical Research Letters, 35 (9), L09 606, doi:10.1029/2008GL033407, URL http: //doi.wiley.com/10.1029/2008GL033407.

Srinivasan, K., J. C. McWilliams, M. J. Molemaker, and R. Barkan, 2019: Submesoscale Vortical Wakes in the Lee of Topography. Journal of Physical Oceanography, 49 (7), 1949-1971, doi: 
10.1175/JPO-D-18-0042.1, URL http://journals.ametsoc.org/doi/10.1175/JPO-D-18-0042.1.

Stone, P. H., 1966: On Non-Geostrophic Baroclinic Stability. Journal of the Atmospheric Sciences, 23 (4), 390-400, doi:10.1175/1520-0469(1966)023〈0390:ONGBS $\rangle$ 2.0.CO; 2, URL http://journals.ametsoc.org/doi/abs/10.1175/1520-0469\%281966\%29023\%3C0390\% 3AONGBS\%3E2.0.CO\%3B2.

Taylor, J. R., and R. Ferrari, 2009: On the equilibration of a symmetrically unstable front via a secondary shear instability. Journal of Fluid Mechanics, 622, 103, doi:10.1017/ S0022112008005272, URL http://www.journals.cambridge.org/abstract_S0022112008005272.

Taylor, J. R., and R. Ferrari, 2010: Buoyancy and Wind-Driven Convection at Mixed Layer Density Fronts. Journal of Physical Oceanography, 40 (6), 1222-1242, doi:10.1175/2010JPO4365. 1, URL http://journals.ametsoc.org/doi/abs/10.1175/2010JPO4365.1.

Thomas, L., and R. Ferrari, 2008: Friction, Frontogenesis, and the Stratification of the Surface Mixed Layer. Journal of Physical Oceanography, 38 (11), 2501-2518, doi:10.1175/ 2008JPO3797.1, URL http://journals.ametsoc.org/doi/abs/10.1175/2008JPO3797.1.

Thomas, L. N., 2005: Destruction of Potential Vorticity by Winds. Journal of Physical Oceanography, 35 (12), 2457-2466, doi:10.1175/JPO2830.1, URL http://journals.ametsoc.org/doi/abs/ 10.1175/JPO2830.1.

Thomas, L. N., 2008: Formation of intrathermocline eddies at ocean fronts by wind-driven destruction of potential vorticity. Dynamics of Atmospheres and Oceans, 45 (3-4), 252273, doi:10.1016/j.dynatmoce.2008.02.002, URL https://linkinghub.elsevier.com/retrieve/pii/ S0377026508000353. 
Thomas, L. N., and J. R. Taylor, 2010: Reduction of the usable wind-work on the general circulation by forced symmetric instability. Geophysical Research Letters, 37 (18), n/a-n/a, doi: 10.1029/2010GL044680, URL http://doi.wiley.com/10.1029/2010GL044680.

Thomas, L. N., J. R. Taylor, E. A. D?Asaro, C. M. Lee, J. M. Klymak, and A. Shcherbina, 2016: Symmetric Instability, Inertial Oscillations, and Turbulence at the Gulf Stream Front. Journal of Physical Oceanography, 46 (1), 197-217, doi:10.1175/JPO-D-15-0008.1, URL http://journals. ametsoc.org/doi/10.1175/JPO-D-15-0008.1.

Thomas, L. N., J. R. Taylor, R. Ferrari, and T. M. Joyce, 2013: Symmetric instability in the Gulf Stream. Deep Sea Research Part II: Topical Studies in Oceanography, 91, 96-110, doi:10.1016/ j.dsr2.2013.02.025, URL http://linkinghub.elsevier.com/retrieve/pii/S0967064513000829.

Thorpe, S. A., 1987: Current and Temperature Variability on the Continental Slope. Philosophical Transactions of the Royal Society of London. Series A, Mathematical and Physical Sciences, 323 (1574), 471-517, URL http://www.jstor.org/stable/38149.

Umlauf, L., W. D. Smyth, and J. N. Moum, 2015: Energetics of Bottom Ekman Layers during Buoyancy Arrest. Journal of Physical Oceanography, 45 (12), 3099-3117, doi:10.1175/ JPO-D-15-0041.1, URL http://journals.ametsoc.org/doi/10.1175/JPO-D-15-0041.1.

Wenegrat, J. O., J. Callies, and L. N. Thomas, 2018: Submesoscale Baroclinic Instability in the Bottom Boundary Layer. Journal of Physical Oceanography, 48 (11), 2571-2592, doi:10.1175/ JPO-D-17-0264.1, URL http://journals.ametsoc.org/doi/10.1175/JPO-D-17-0264.1.

Wenegrat, J. O., and M. J. McPhaden, 2016: Wind, Waves, and Fronts: Frictional Effects in a Generalized Ekman Model. Journal of Physical Oceanography, 46 (2), 371-394, doi:10.1175/ JPO-D-15-0162.1, URL http://journals.ametsoc.org/doi/abs/10.1175/JPO-D-15-0162.1. 
Yankovsky, E., and S. Legg, 2019: Symmetric and Baroclinic Instability in Dense Shelf Overflows.

Journal of Physical Oceanography, 49 (1), 39-61, doi:10.1175/JPO-D-18-0072.1, URL http: //journals.ametsoc.org/doi/10.1175/JPO-D-18-0072.1. 
LIST OF TABLES

Table 1. Summary of numerical simulations. All simulations are run with $f=10^{-4}$ $\mathrm{s}^{-1}$, and an interior velocity of $V_{\infty}=0.1 \mathrm{~m} \mathrm{~s}^{-1}$ except as indicated by a $\neq$ where an increased velocity of $V_{\infty}=0.2 \mathrm{~m} \mathrm{~s}^{-1}$ was used. Simulations that were dominated by convective instability are indicated by the $\dagger$ symbol (section $3 \mathrm{c}$ ). 
TABLE 1. Summary of numerical simulations. All simulations are run with $f=10^{-4} \mathrm{~s}^{-1}$, and an interior velocity of $V_{\infty}=0.1 \mathrm{~m} \mathrm{~s}^{-1}$ except as indicated by a $\neq$ where an increased velocity of $V_{\infty}=0.2 \mathrm{~m} \mathrm{~s}^{-1}$ was used.

Simulations that were dominated by convective instability are indicated by the $\dagger$ symbol (section $3 \mathrm{c}$ ).

\begin{tabular}{|c|c|c|c|c|}
\hline Name & $\begin{array}{l}\text { Interior Stratification } \\
\qquad N_{\infty}^{2}\left(\mathrm{~s}^{-2}\right)\end{array}$ & $\begin{array}{c}\text { Slope Angle } \\
\theta\end{array}$ & $\begin{array}{l}\text { Slope Burger Number } \\
\qquad S_{\infty}=N_{\infty} \tan \theta / f\end{array}$ & $\begin{array}{l}\text { Model Configuration } \\
L_{x} \times L_{z}-\text { Run duration }\end{array}$ \\
\hline \multirow{3}{*}{ CI-1 } & $10^{-5}$ & 0.1 & 3.2 & $1 \mathrm{~km} \times 200 \mathrm{~m}-40$ days \\
\hline & $10^{-5}$ & 0.05 & 1.6 & $1 \mathrm{~km} \times 200 \mathrm{~m}-40$ days \\
\hline & $10^{-6}$ & 0.1 & 1 & $1 \mathrm{~km} \times 300 \mathrm{~m}-30$ days \\
\hline SI-1 & $10^{-5}$ & 0.02 & 0.6 & $1 \mathrm{~km} \times 200 \mathrm{~m}-40$ days \\
\hline \multirow[t]{3}{*}{$\ddagger$} & $10^{-5}$ & 0.02 & 0.6 & $2 \mathrm{~km} \times 300 \mathrm{~m}-15$ days \\
\hline & $10^{-6}$ & 0.06 & 0.6 & $1 \mathrm{~km} \times 200 \mathrm{~m}-15$ days \\
\hline & $5 \times 10^{-6}$ & 0.02 & 0.45 & $1 \mathrm{~km} \times 200 \mathrm{~m}-40$ days \\
\hline$\dagger$ & $10^{-6}$ & 0.01 & 0.1 & $1 \mathrm{~km} \times 200 \mathrm{~m}-40$ days \\
\hline$\dagger$ & $10^{-7}$ & 0.02 & 0.06 & $1 \mathrm{~km} \times 200 \mathrm{~m}-40$ days \\
\hline CONV-1 $1^{\dagger}$ & $10^{-7}$ & 0.005 & 0.02 & $1 \mathrm{~km} \times 200 \mathrm{~m}-40$ days \\
\hline
\end{tabular}




\section{LIST OF FIGURES}

Fig. 1. Schematic of the numerical model domain (panel A), with initial condition consisting of a uniformly stratified interior (with buoyancy contours indicated by thin black lines) and barotropic interior flow over a sloping lower boundary. An example of the adjustment of the boundary layer towards thermal wind balance is shown in panel $\mathrm{B}$, where downslope Ekman transport generates thermal wind shear in the bottom boundary layer, reducing the near-bottom velocities. The rotated coordinate system is also shown, with standard nonrotated coordinates denoted using the carat notation.

Fig. 2. Overview of the evolution of simulation SI-1 (with parameters as given in table 1). From top to bottom the panels give the across-slope velocity $(u)$, the total along-slope velocity $\left(v_{T}=v+V_{\infty}\right)$, the vertical buoyancy gradient $\left(N^{2}\right)$, and the PV $(q)$. All values are averaged in the across-slope $(x)$ direction, and normalized as indicated in each plot. The evolution of the low PV layer depth $H$, as predicted by (27), is shown in the bottom panel in black.

Fig. 3. Snapshot of the across-slope velocity field ( $u$, color scale) from day 12 of run SI-1. The banded velocity structure is typical of symmetric instability, where the fastest growing mode is oriented along isopycnals (black contours). The height of the low PV layer ( $H$, section $3 \mathrm{~b})$ and the convective layer ( $h$, section $3 \mathrm{c}$ ) as determined from the numerical solutions are indicted along the right ordinate by the large and small triangles, respectively.

Fig. 4. Cross-frontal section detailing the spatial distribution of the fastest growing instability type predicted from linear theory for run SI-1 on day 12 (as in figure 3). Parameter space is divided into mixed centrifugal-symmetric (CI-SI), symmetric (SI), gravitational (GRAV), and mixed gravitational-symmetric (GRAV-SI) following the balanced Richardson number criteria laid out in Thomas et al. (2013, as discussed in section 2b). Isopycnals are shown with black contours. All fields are averaged over a 4 hour period, and the height of the low PV layer ( $H$, section $3 b)$ and the convective layer ( $h$, section $3 c)$ as determined from the numerical solutions are indicted along the right ordinate by the large and small triangles, respectively.

Fig. 5. As in figure 2, but for simulation CI-1 (with parameters as given in table 1). From top to bottom the panels give the across-slope velocity $(u$, note the reduced color scale from figure 2 ), the total along-slope velocity $\left(v_{T}=v+V_{\infty}\right)$, the vertical buoyancy gradient $\left(N^{2}\right)$, and the PV $(q)$. All values are averaged in the across-slope $(x)$ direction, and normalized as indicated in each plot. The evolution of the low PV layer depth $H$, as predicted by (27), is shown in the bottom panel in black.

Fig. 6. As in figure 3, but for run CI-1 on day 12.

Fig. 7. Cross-frontal section detailing the spatial distribution of the fastest growing instability type predicted from linear theory for run CI-1 on day 12 (as in figure 6). See the caption of figure 4 for definitions.

Fig. 8. Dominant terms in the horizontally averaged $(x-y)$ momentum budgets for simulation SI-1, averaged over days 11-13. The across-slope momentum budget, (7), is largely in a geostrophic balance between buoyancy perturbations and Coriolis accelerations, with some additional contribution from the turbulent momentum flux divergence (left panel). The along-slope momentum budget is in approximate Ekman balance, with Coriolis accelerations balancing the turbulent momentum flux divergence (right panel). The height of the low PV layer ( $H$, large triangle) and the convective layer $(h$, small triangle) are indicted along the right ordinate in each plot. 
Fig. 9. Terms from the slope-normal PV flux equation, (19), averaged horizontally $(x)$ and over days 11-13 for simulation SI-1. A) Away from the bottom the sum of the two flux terms is an approximately linear function of $H$, as required for $\left\langle q^{\prime} w^{\prime}\right\rangle$ to be non-divergent over the boundary layer (section $3 \mathrm{a}$ ). The scaling for the PV flux magnitude, $\left(1+S_{\infty}^{2}\right) E B F_{s}$, is also shown (dashed black line). B) The same flux terms (as defined in the legend) decomposed by across-slope wavelength, where long wavelength motions $\left(\lambda_{x}>100 \mathrm{~m}\right.$, solid lines) are associated with the instability overturning cells, and small-scale motions $\left(\lambda_{x}<100 \mathrm{~m}\right.$, dashed lines) are associated with turbulence. The height of the low PV layer $(H$, large triangle) and the convective layer ( $h$, small triangle) are indicted along the right ordinate in each plot.

Fig. 10. Comparison of the convective layer depth predicted from (33), $h$, and the depth determined from the numerical simulations, $h_{N U M}$. The squared correlation coefficient is shown in the plot title. For the numerical simulations the height of the convective layer is determined as the lowest height where ${\overline{\left\langle w^{\prime} b^{\prime}\right\rangle}}^{T}<0$, where the overbar indicates averaging over 1 inertial period and turbulent motions are defined as having a cross-slope wavelength of $\lambda_{x}<100 \mathrm{~m}$. The height of the low PV layer is determined from the numerical simulations as the height at which $\overline{\langle q\rangle}=0.5 f N_{\infty}^{2}$, ie the height at which the PV reaches $50 \%$ of the interior PV. Instances where the bottom diffusive flux of perturbation buoyancy $\left(\kappa N_{\infty}^{2}\right)$ exceeds $E B F_{s} /\left(1+S_{\infty}^{2}\right)$ are excluded for consistency with the assumptions of section 3 (and appendix).

Fig. 11. As in figure 3, but for run CONV-1 on day 12. In this simulation, where $L_{s} / H \gg 1$, SI/CI does not develop, and convective turbulence keeps the boundary layer unstratified.

Fig. 12. Cross-frontal section detailing the spatial distribution of the fastest growing instability type predicted from linear theory for run CONV-1 on day 12 (as in figure 11). See the caption of figure 4 for definitions.

Fig. 13. Comparison of the true lateral shear production (LSP) to the true vertical shear production (VSP) for run CI-1 (left) and SI-1 (right). Profiles are averaged over days 4-6. In simulation CI-1, where $S_{\infty}>1$, LSP dominates outside a thin-near boundary region, whereas simulation SI-1 $\left(S_{\infty}<1\right)$ is dominated by VSP everywhere, consistent with the expectation from (40) and (41). The height of the low PV layer ( $H$, large triangle) and the convective layer $(h$, small triangle) for each simulation are indicted along the right ordinates.

Fig. 14. Cumulative energy budget over the first 15 days of simulation SI-1, formed by taking the vertical integral of (35), and then integrating in time.

Fig. 15. Comparison between eddy kinetic energy production and dissipation. Panel a and b show slope-normal profiles of shear production (SP), vertical buoyancy production (VBP), and dissipation (DISS) from simulation CI-1 (panel a) and SI-1 (panel b), averaged over days 4-6. Terms are defined as in (35). Also shown is minus the $E B F_{s}$ (gray dashed-dot line), and the scaling for the dissipation (black dashed line) given in equation (47). Panel c shows a comparison between the scaling for the depth-integrated dissipation rate and the depthintegrated geostrophic shear production plus the buoyancy production across all simulations. The squared correlation coefficient is shown in the plot title. Note that here these terms are evaluated using the approximate form given by (46), ie. $G S P_{s}=-\left\langle v^{\prime} w^{\prime}\right\rangle N_{\infty}^{2} \theta / f\left(1+S_{\infty}^{2}\right)$ and $V B P_{s}=\left\langle w^{\prime} b^{\prime}\right\rangle /\left(1+S_{\infty}^{2}\right)$. The simulation with $V_{\infty}=0.2 \mathrm{~m} \mathrm{~s}^{-1}$ is off the scale shown on this plot, however it also closely follows the 1-1 line.

Fig. 16. Evolution of the average along-slope bottom stress, $\left\langle\tau^{y}\right\rangle$, for all simulations (table 1). The top panel shows the evolution of the stress as a function of time. The bottom panel shows the stress evolution with time normalized by the Ekman adjustment timescale, which collapses 
all simulations to a single curve (similar to the results for 1D simulations shown in Brink and Lentz 2010). Simulations with active SI/CI (circular markers) are normalized using the timescale (49), and simulations where convection dominates (diamond markers) are normalized using (50). In both plots the stress is averaged over 12 hour periods, and normalized by the maximum value for each simulation. 


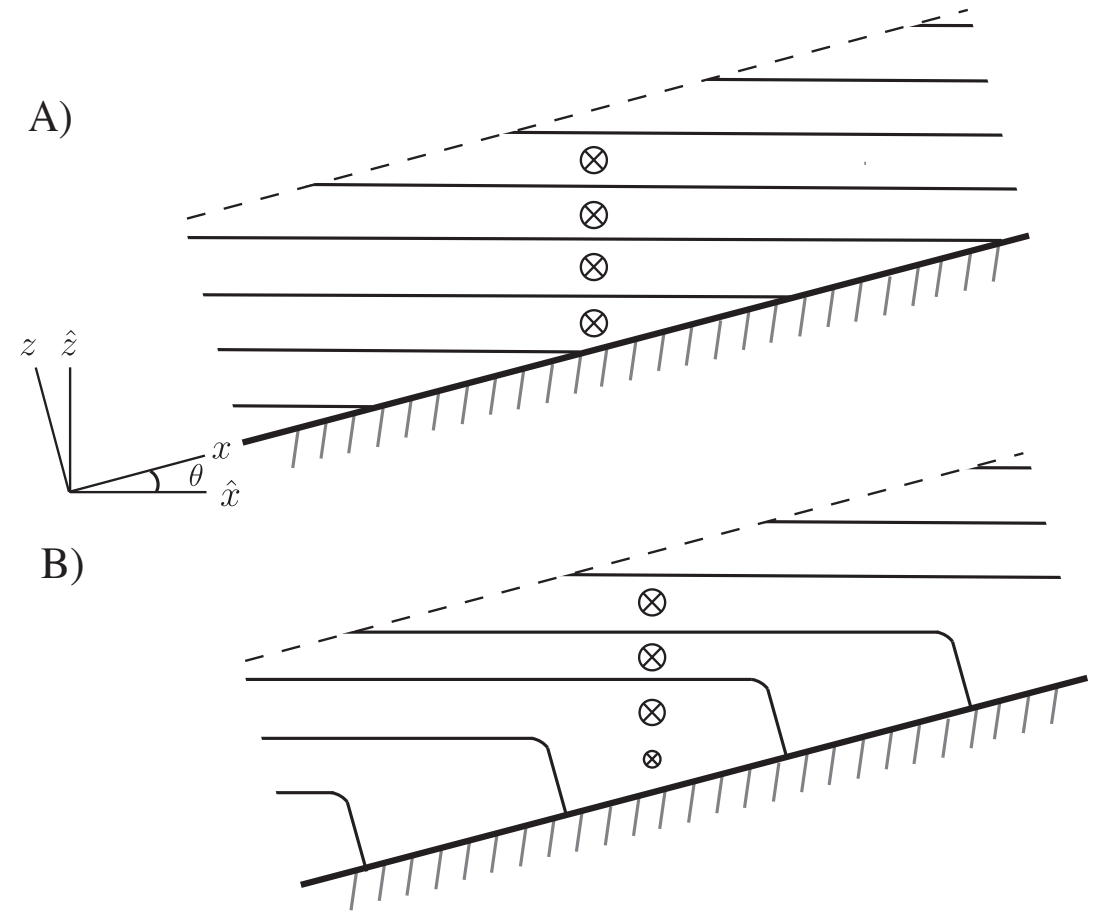

FIG. 1. Schematic of the numerical model domain (panel A), with initial condition consisting of a uniformly stratified interior (with buoyancy contours indicated by thin black lines) and barotropic interior flow over a sloping lower boundary. An example of the adjustment of the boundary layer towards thermal wind balance is shown in panel B, where downslope Ekman transport generates thermal wind shear in the bottom boundary layer, reducing the near-bottom velocities. The rotated coordinate system is also shown, with standard nonrotated coordinates denoted using the carat notation. 

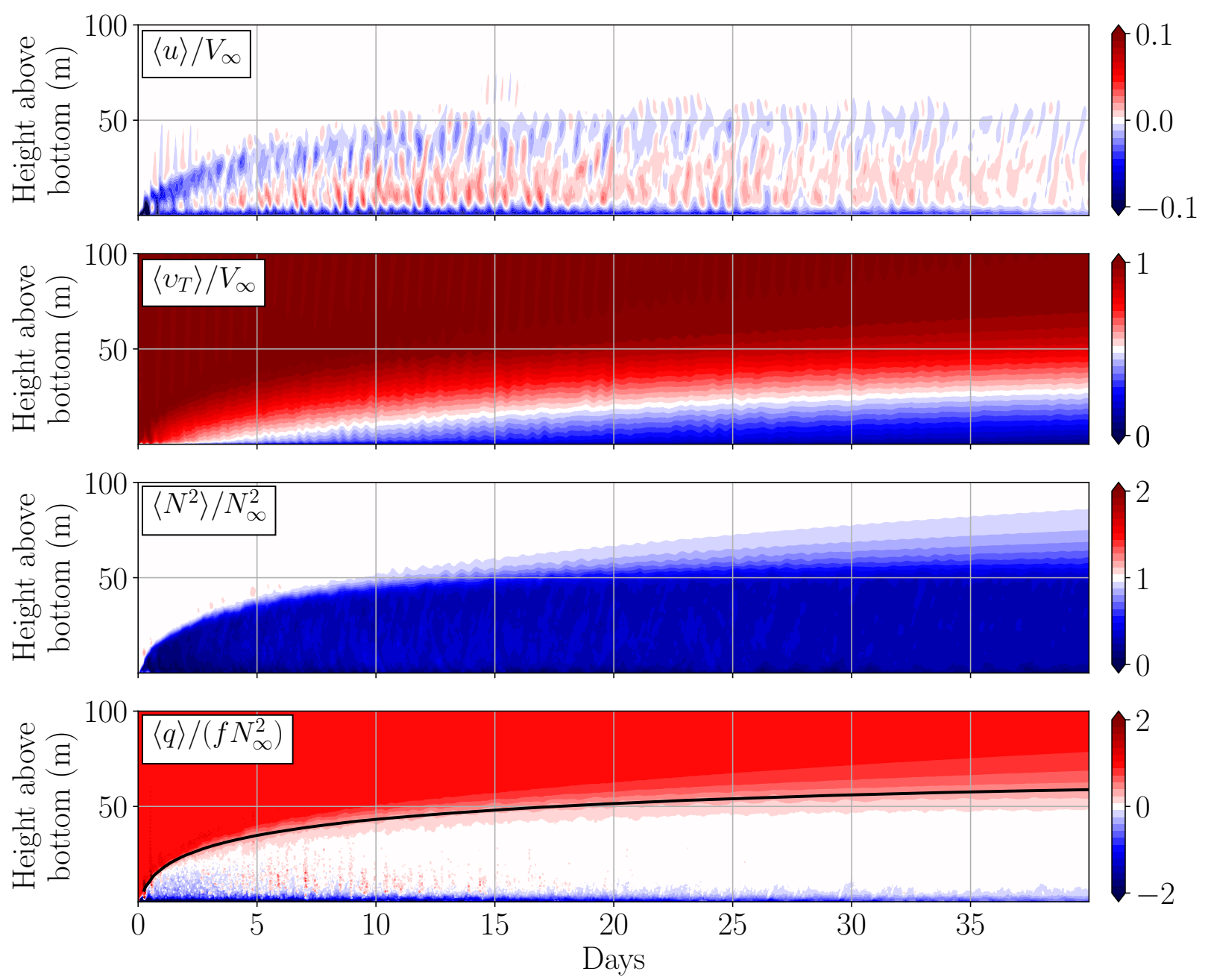

FIG. 2. Overview of the evolution of simulation SI-1 (with parameters as given in table 1). From top to bottom the panels give the across-slope velocity $(u)$, the total along-slope velocity $\left(v_{T}=v+V_{\infty}\right)$, the vertical buoyancy gradient $\left(N^{2}\right)$, and the PV $(q)$. All values are averaged in the across-slope $(x)$ direction, and normalized as indicated in each plot. The evolution of the low PV layer depth $H$, as predicted by (27), is shown in the bottom panel in black. 


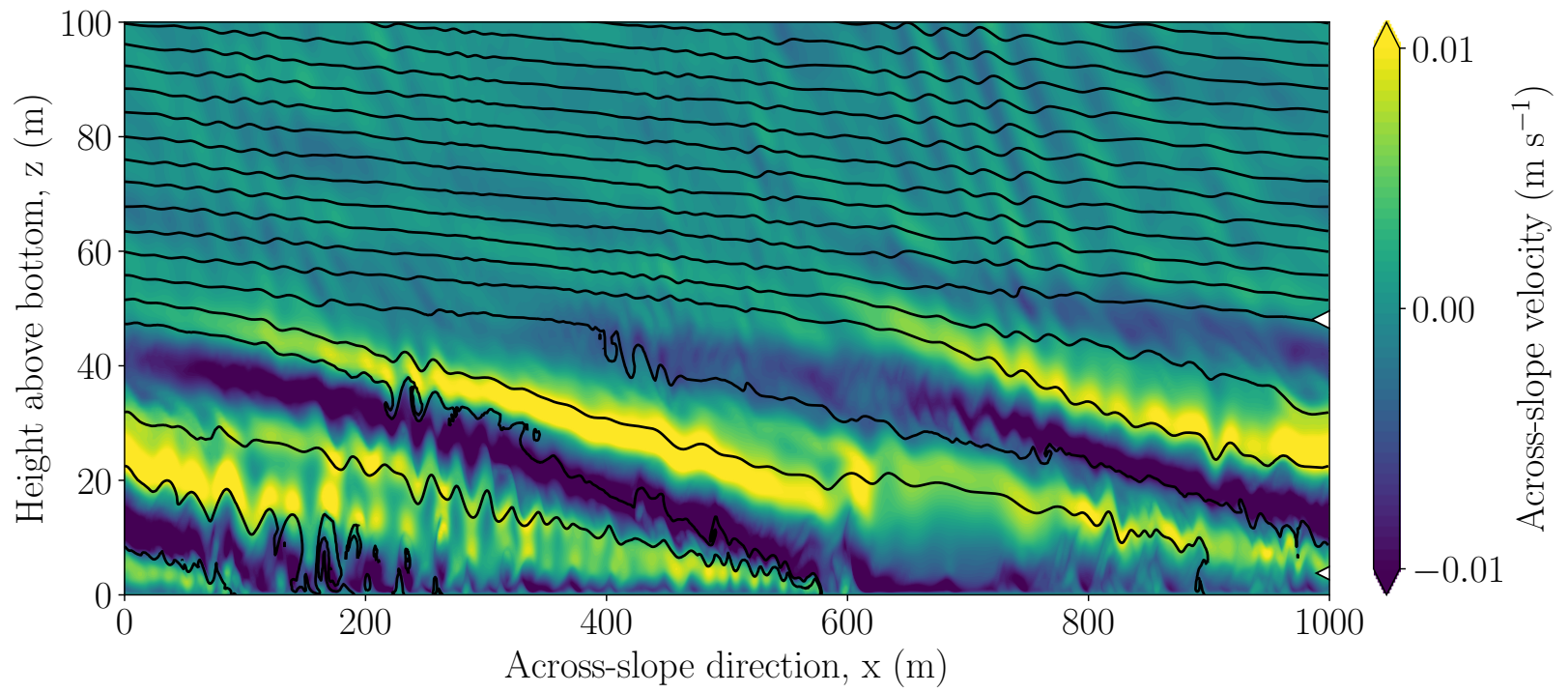

FIG. 3. Snapshot of the across-slope velocity field ( $u$, color scale) from day 12 of run SI-1. The banded velocity structure is typical of symmetric instability, where the fastest growing mode is oriented along isopycnals (black contours). The height of the low PV layer $(H$, section $3 \mathrm{~b})$ and the convective layer ( $h$, section $3 \mathrm{c})$ as determined from the numerical solutions are indicted along the right ordinate by the large and small triangles, respectively. 


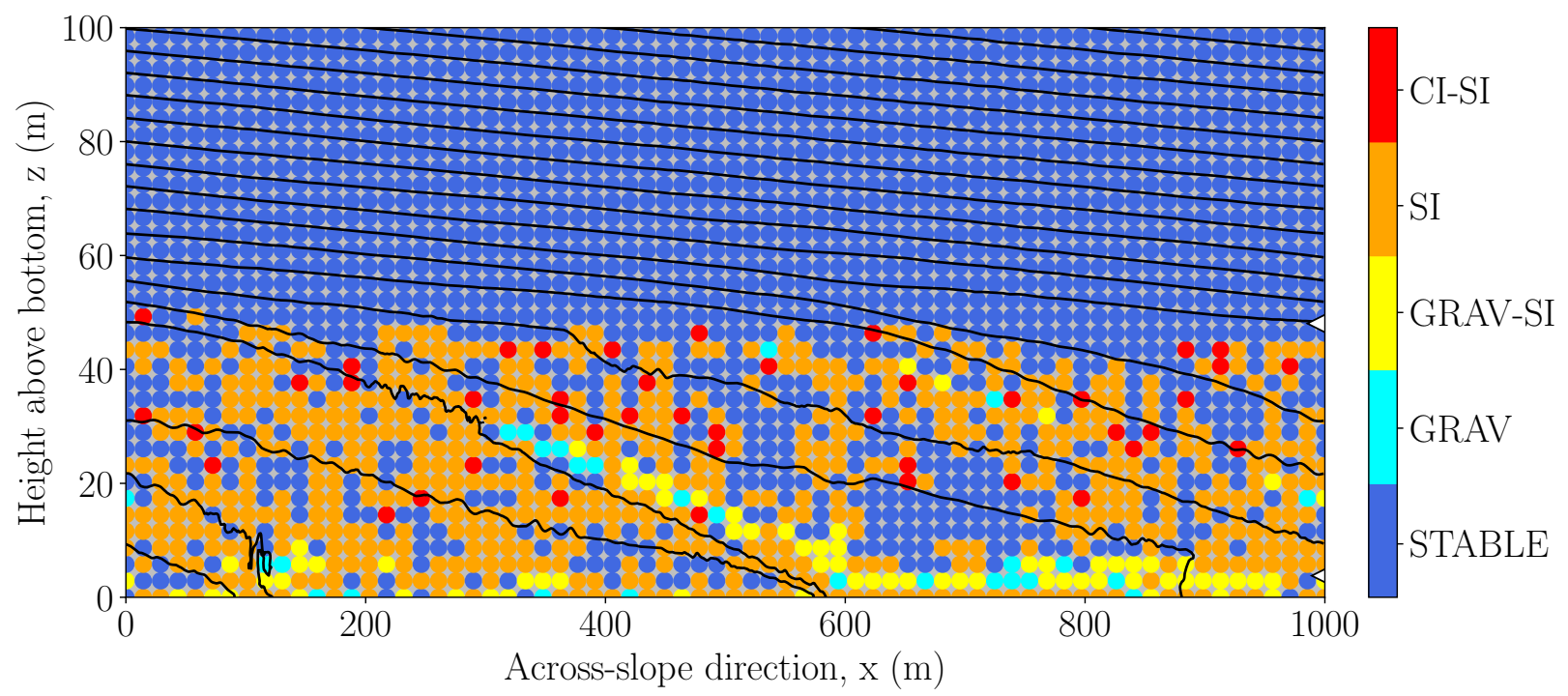

FIG. 4. Cross-frontal section detailing the spatial distribution of the fastest growing instability type predicted from linear theory for run SI-1 on day 12 (as in figure 3). Parameter space is divided into mixed centrifugalsymmetric (CI-SI), symmetric (SI), gravitational (GRAV), and mixed gravitational-symmetric (GRAV-SI) following the balanced Richardson number criteria laid out in Thomas et al. (2013, as discussed in section 2b). Isopycnals are shown with black contours. All fields are averaged over a 4 hour period, and the height of the low PV layer $(H$, section $3 b)$ and the convective layer ( $h$, section $3 c)$ as determined from the numerical solutions are indicted along the right ordinate by the large and small triangles, respectively. 

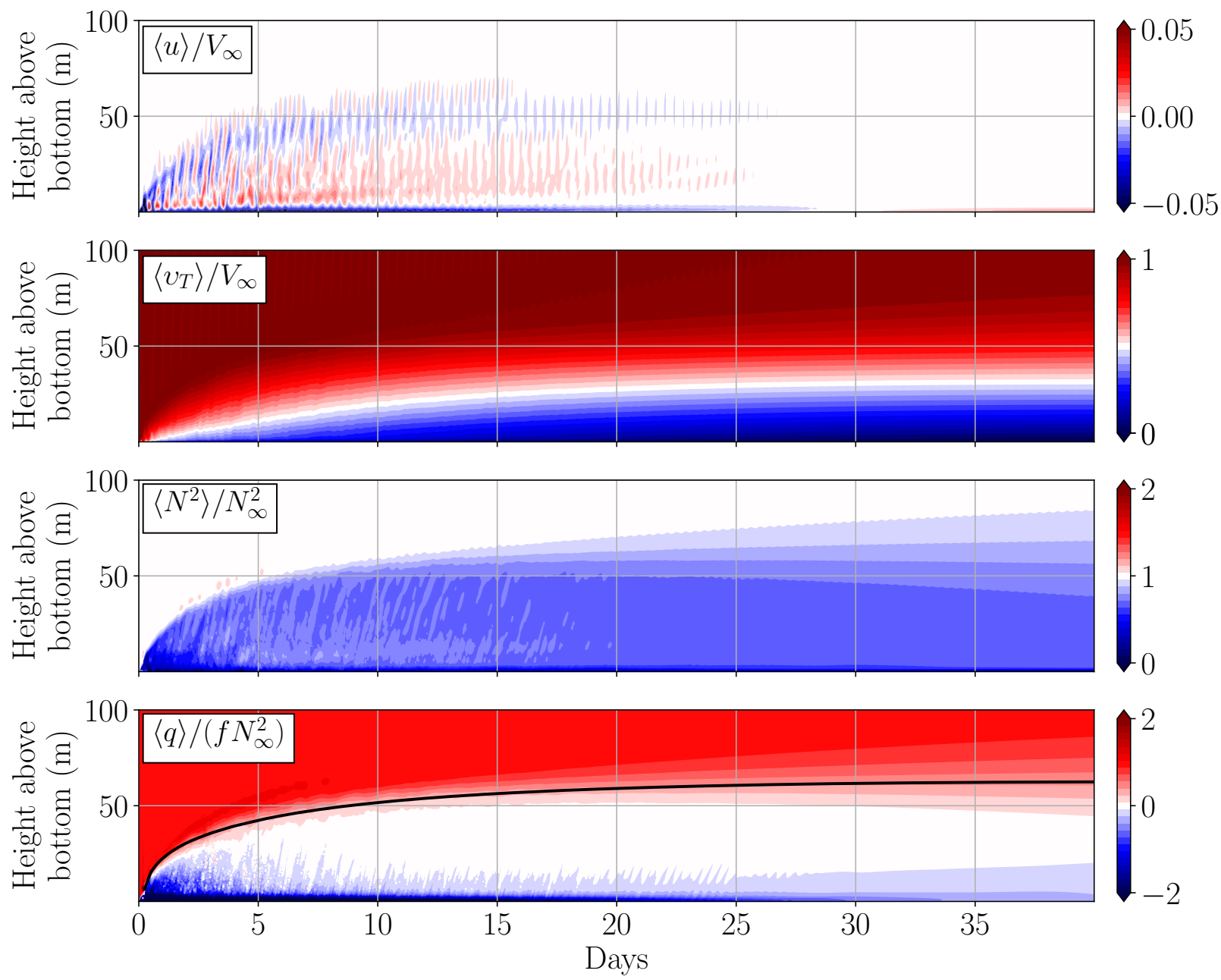

FIG. 5. As in figure 2, but for simulation CI-1 (with parameters as given in table 1). From top to bottom the panels give the across-slope velocity ( $u$, note the reduced color scale from figure 2$)$, the total along-slope velocity $\left(v_{T}=v+V_{\infty}\right)$, the vertical buoyancy gradient $\left(N^{2}\right)$, and the PV $(q)$. All values are averaged in the across-slope $(x)$ direction, and normalized as indicated in each plot. The evolution of the low PV layer depth $H$, as predicted by (27), is shown in the bottom panel in black. 


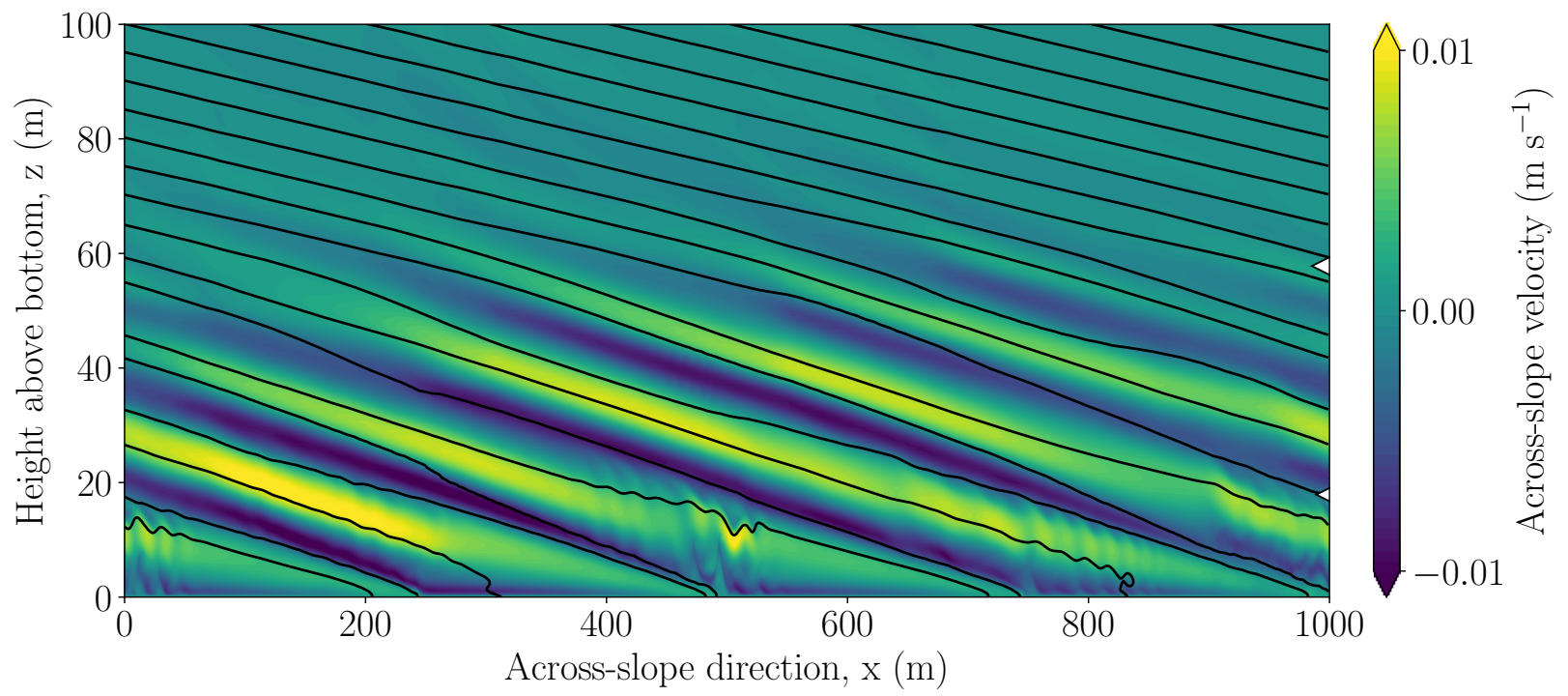

FIG. 6. As in figure 3, but for run CI-1 on day 12. 


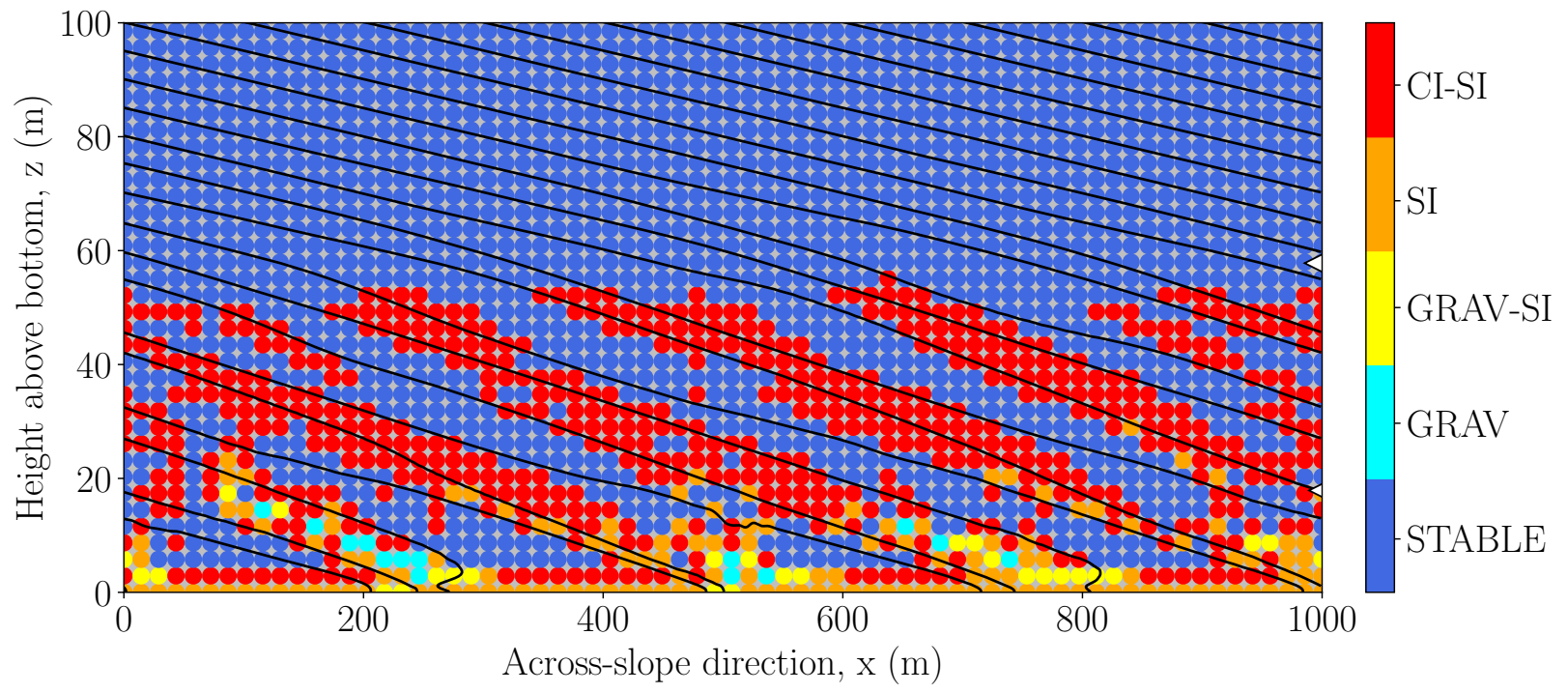

FIG. 7. Cross-frontal section detailing the spatial distribution of the fastest growing instability type predicted ${ }_{850}$ from linear theory for run CI-1 on day 12 (as in figure 6). See the caption of figure 4 for definitions. 

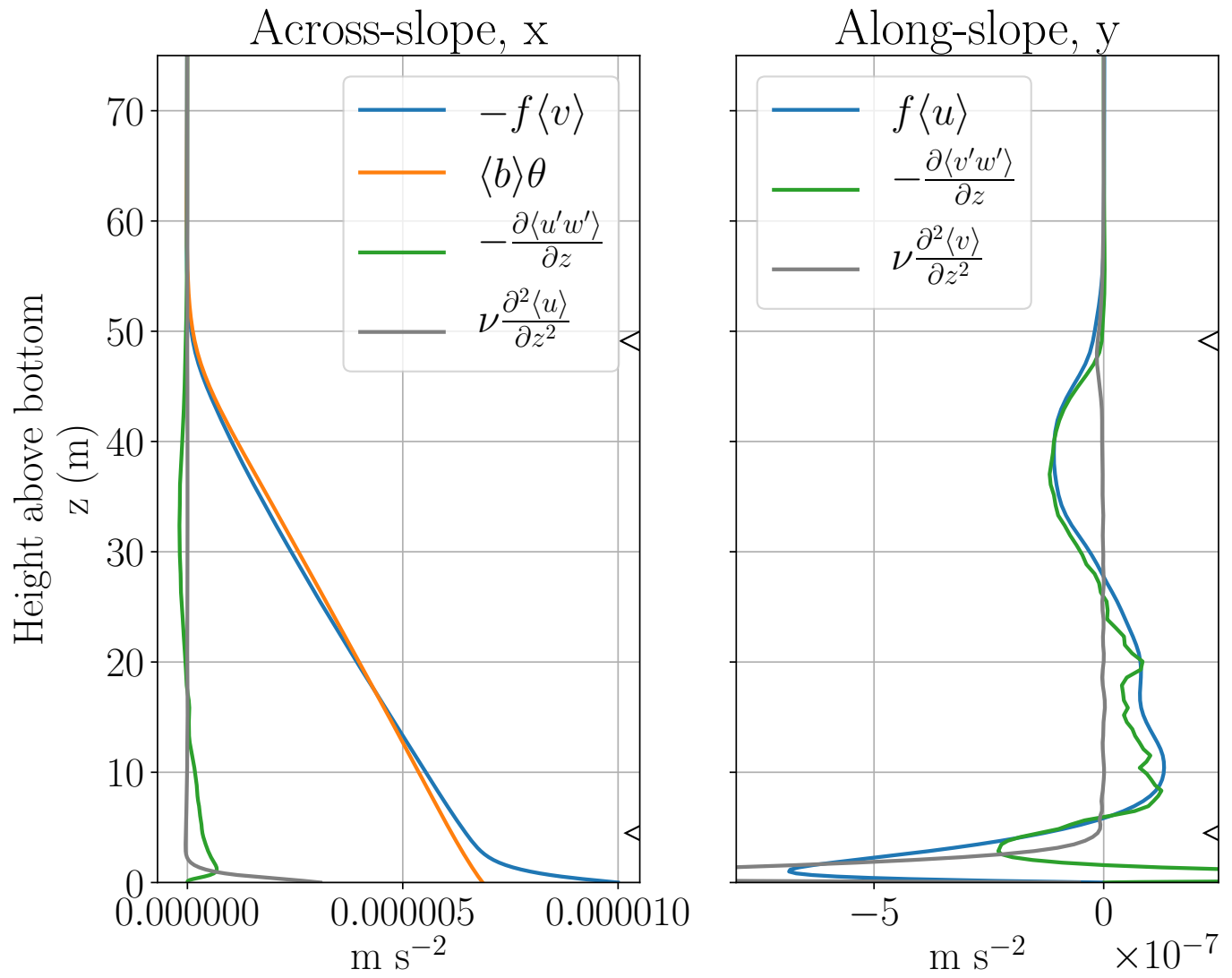

FIG. 8. Dominant terms in the horizontally averaged $(x-y)$ momentum budgets for simulation SI-1, averaged over days 11-13. The across-slope momentum budget, (7), is largely in a geostrophic balance between buoyancy perturbations and Coriolis accelerations, with some additional contribution from the turbulent momentum flux divergence (left panel). The along-slope momentum budget is in approximate Ekman balance, with Coriolis accelerations balancing the turbulent momentum flux divergence (right panel). The height of the low PV layer ( $H$, large triangle) and the convective layer ( $h$, small triangle) are indicted along the right ordinate in each plot. 

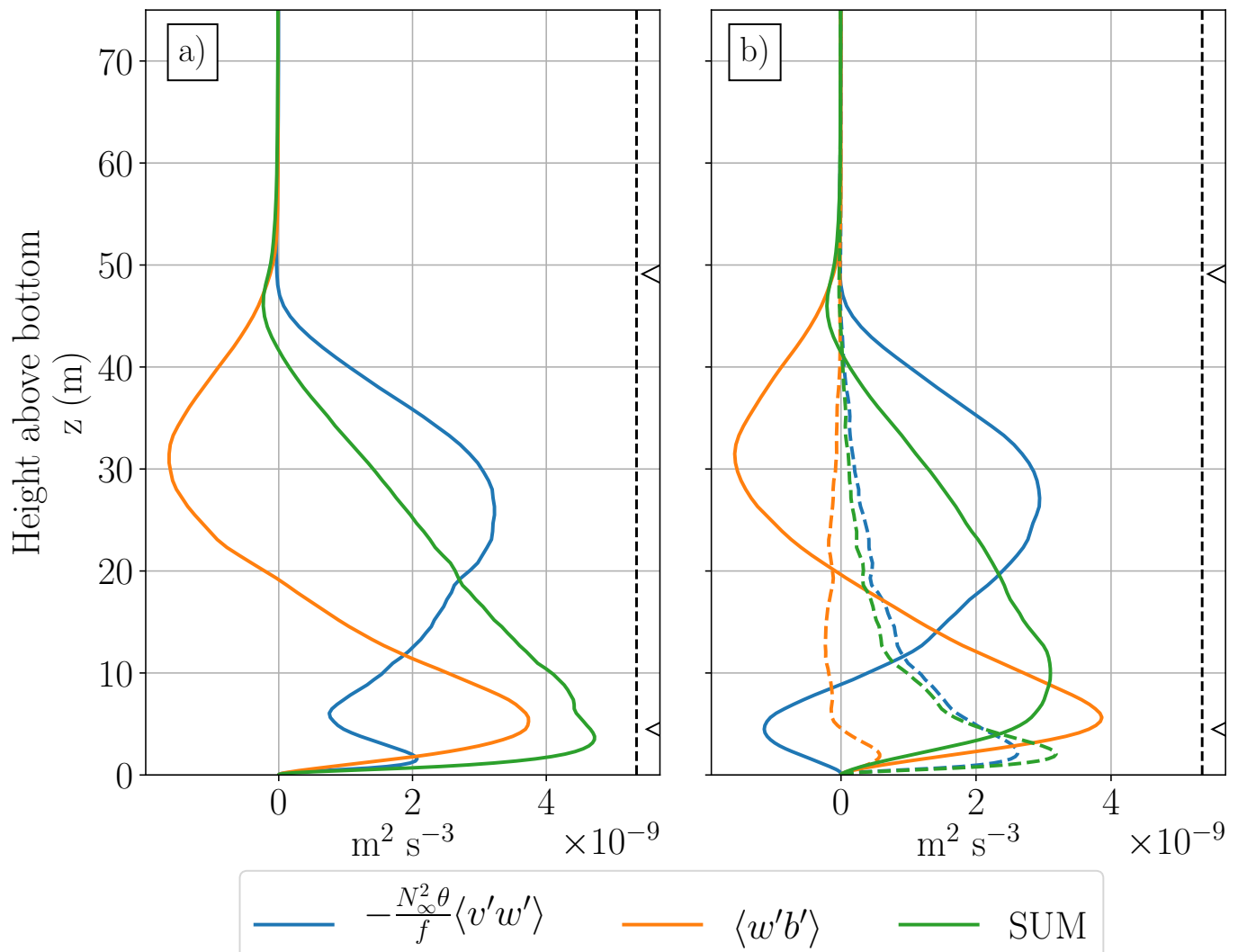

$\left\langle w^{\prime} b^{\prime}\right\rangle$

\section{SUM}

FIG. 9. Terms from the slope-normal PV flux equation, (19), averaged horizontally $(x)$ and over days 11-13 for simulation SI-1. A) Away from the bottom the sum of the two flux terms is an approximately linear function of $H$, as required for $\left\langle q^{\prime} w^{\prime}\right\rangle$ to be non-divergent over the boundary layer (section 3a). The scaling for the PV flux magnitude, $\left(1+S_{\infty}^{2}\right) E B F_{s}$, is also shown (dashed black line). B) The same flux terms (as defined in the legend) decomposed by across-slope wavelength, where long wavelength motions $\left(\lambda_{x}>100 \mathrm{~m}\right.$, solid lines) are associated with the instability overturning cells, and small-scale motions ( $\lambda_{x}<100 \mathrm{~m}$, dashed lines) are associated with turbulence. The height of the low PV layer $(H$, large triangle) and the convective layer $(h$, small triangle) are indicted along the right ordinate in each plot. 


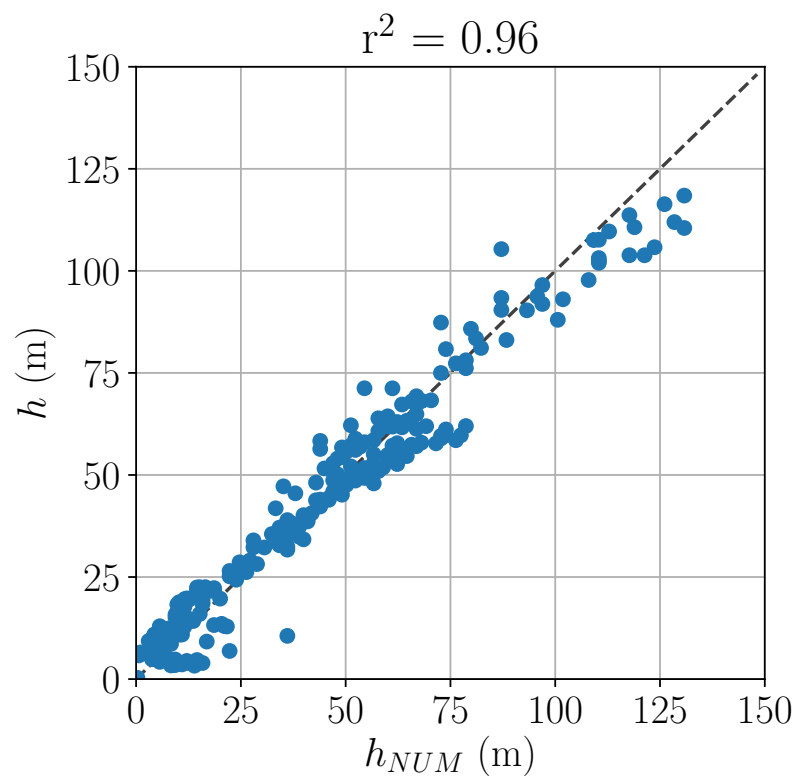

FIG. 10. Comparison of the convective layer depth predicted from (33), $h$, and the depth determined from the numerical simulations, $h_{N U M}$. The squared correlation coefficient is shown in the plot title. For the numerical simulations the height of the convective layer is determined as the lowest height where ${\overline{\left\langle w^{\prime} b^{\prime}\right\rangle}}^{T}<0$, where the overbar indicates averaging over 1 inertial period and turbulent motions are defined as having a cross-slope wavelength of $\lambda_{x}<100 \mathrm{~m}$. The height of the low PV layer is determined from the numerical simulations as the height at which $\overline{\langle q\rangle}=0.5 f N_{\infty}^{2}$, ie the height at which the PV reaches $50 \%$ of the interior PV. Instances where the bottom diffusive flux of perturbation buoyancy $\left(\kappa N_{\infty}^{2}\right)$ exceeds $E B F_{S} /\left(1+S_{\infty}^{2}\right)$ are excluded for consistency with the assumptions of section 3 (and appendix). 


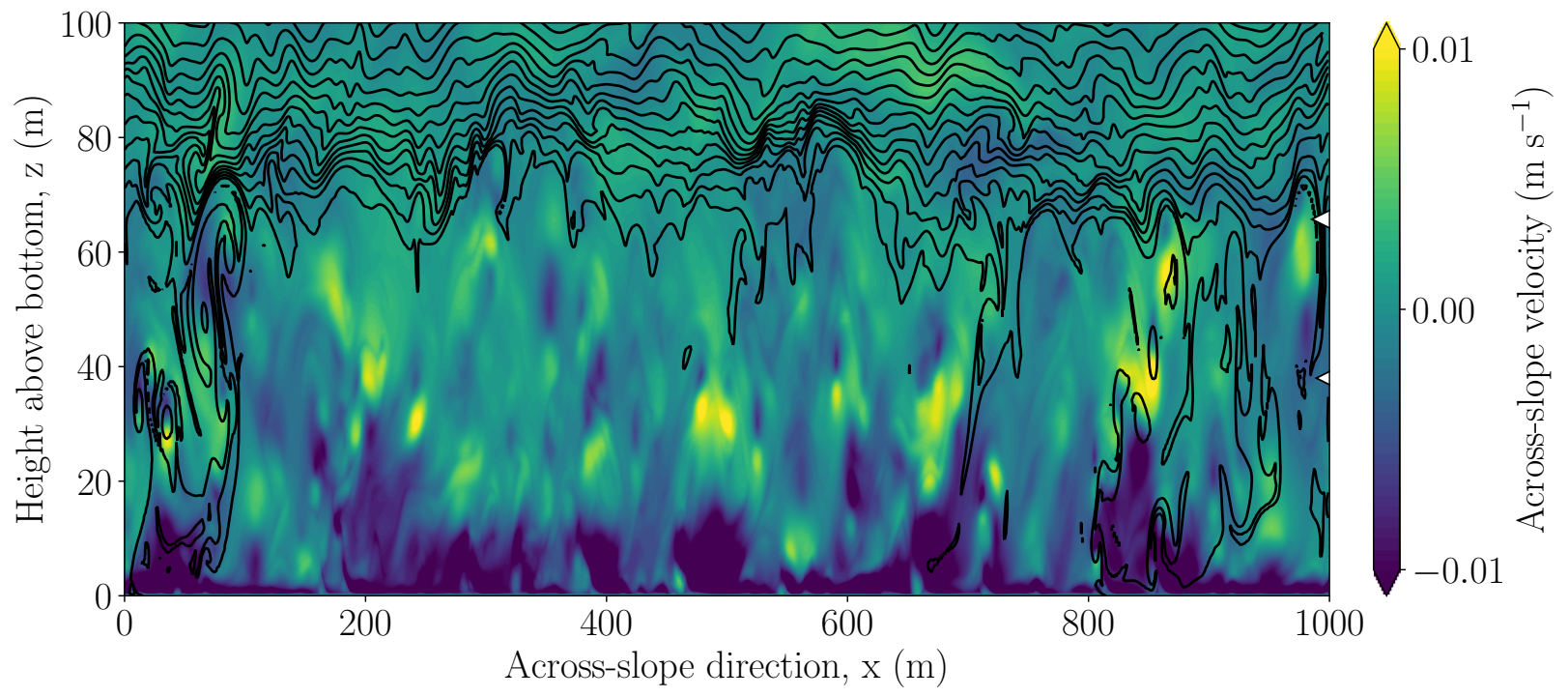

FIG. 11. As in figure 3, but for run CONV-1 on day 12. In this simulation, where $L_{S} / H \gg 1$, SI/CI does not develop, and convective turbulence keeps the boundary layer unstratified. 


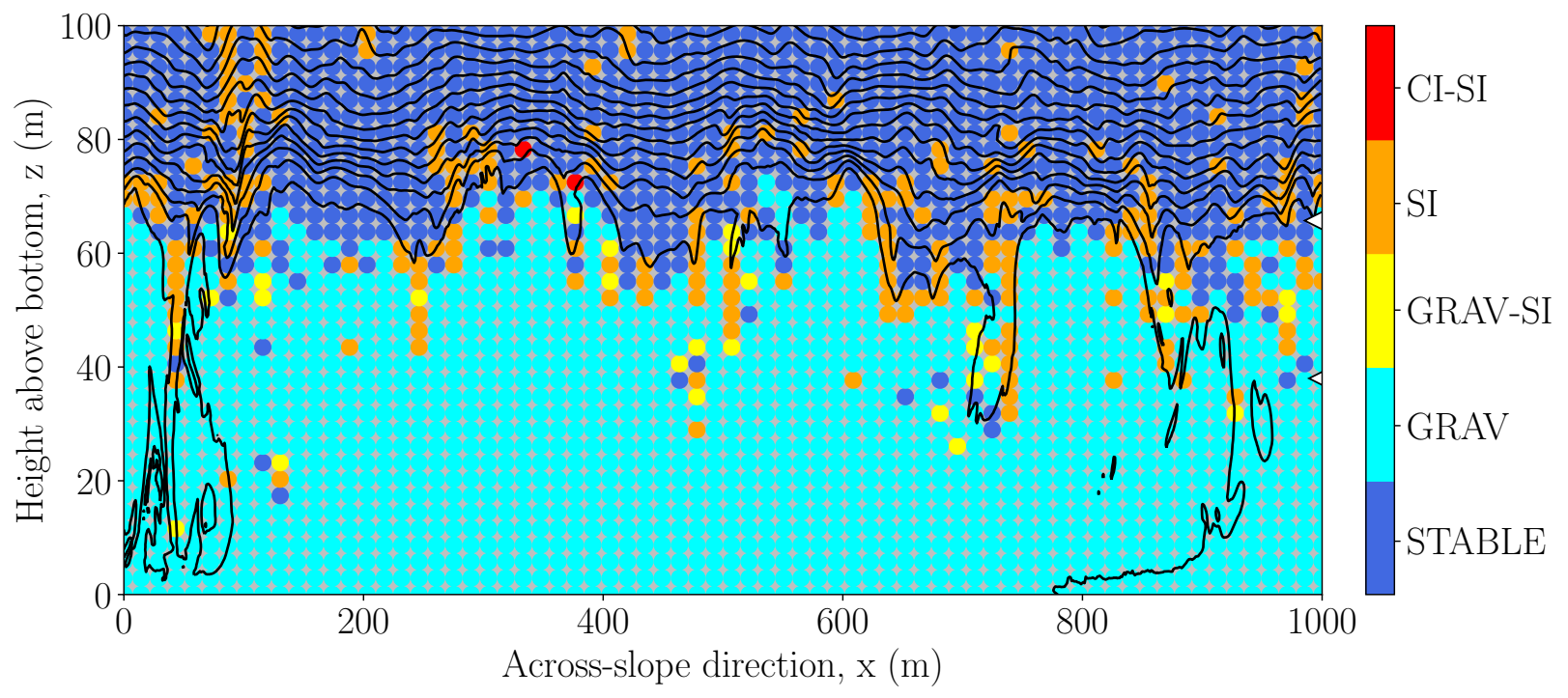

FIG. 12. Cross-frontal section detailing the spatial distribution of the fastest growing instability type predicted from linear theory for run CONV-1 on day 12 (as in figure 11). See the caption of figure 4 for definitions. 


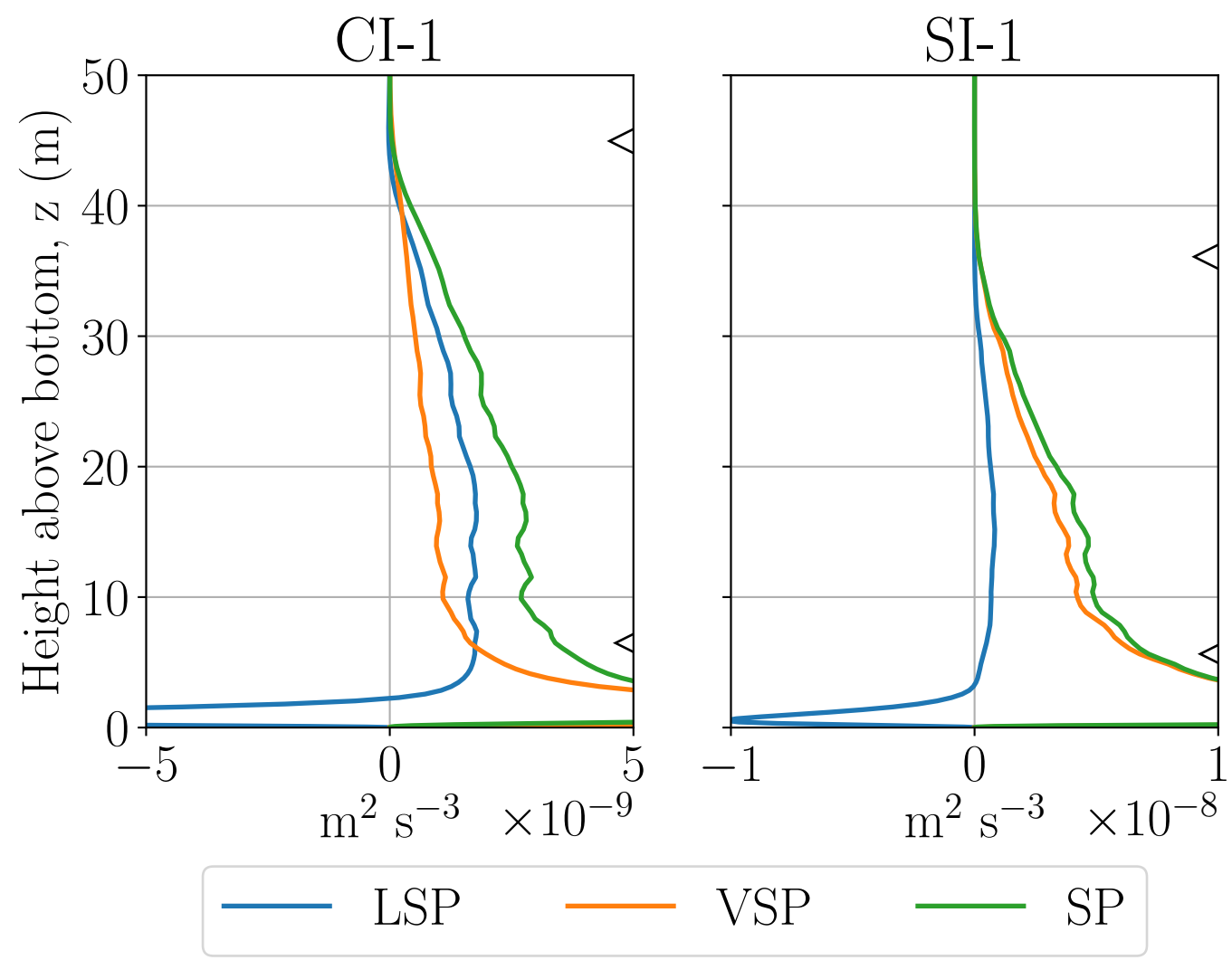

FIG. 13. Comparison of the true lateral shear production (LSP) to the true vertical shear production (VSP) for run CI-1 (left) and SI-1 (right). Profiles are averaged over days 4-6. In simulation CI-1, where $S_{\infty}>1$, LSP dominates outside a thin-near boundary region, whereas simulation SI-1 $\left(S_{\infty}<1\right)$ is dominated by VSP everywhere, consistent with the expectation from (40) and (41). The height of the low PV layer $(H$, large triangle) and the convective layer ( $h$, small triangle) for each simulation are indicted along the right ordinates. 


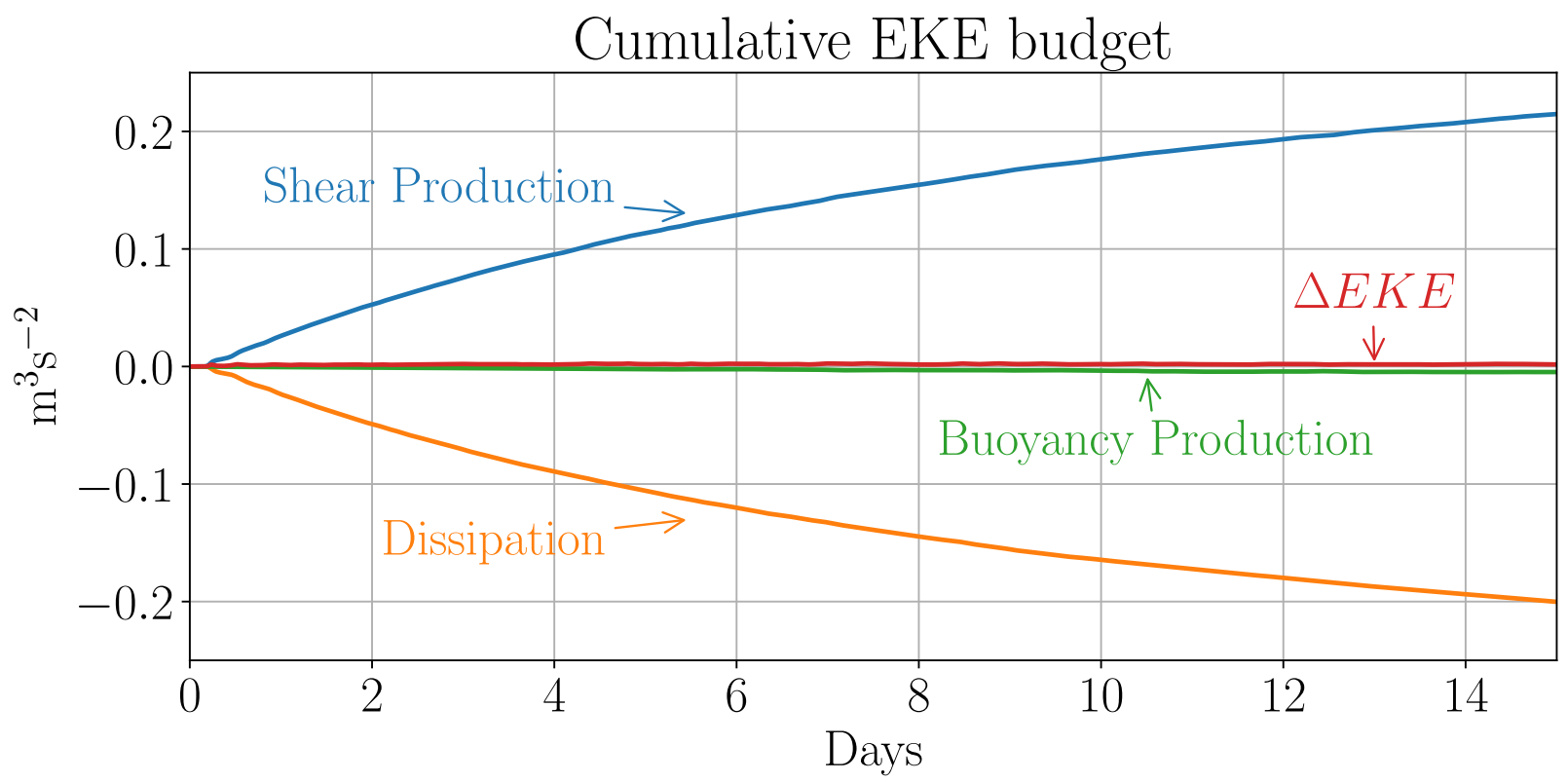

FIG. 14. Cumulative energy budget over the first 15 days of simulation SI-1, formed by taking the vertical integral of (35), and then integrating in time. 

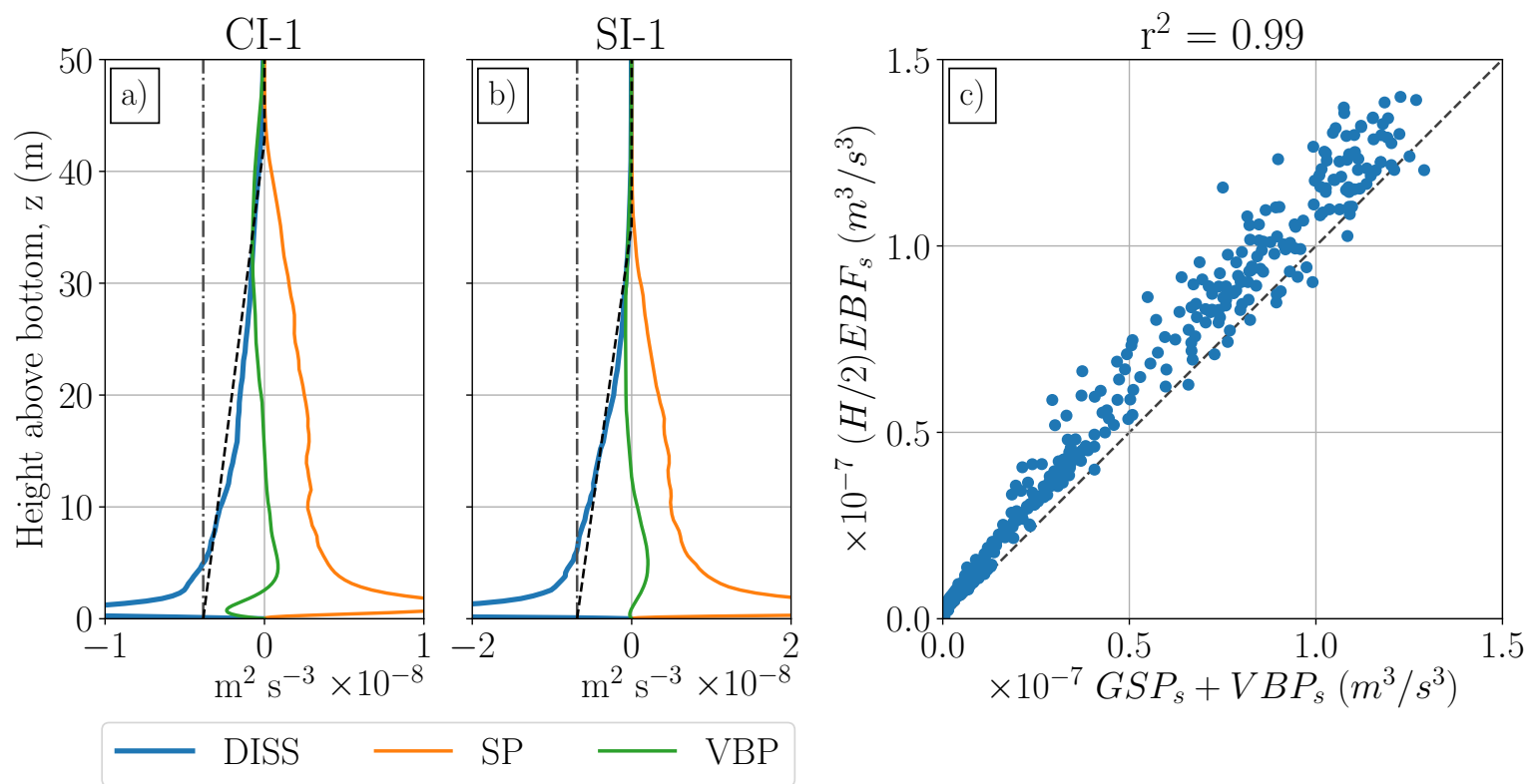

FIG. 15. Comparison between eddy kinetic energy production and dissipation. Panel a and b show slopenormal profiles of shear production (SP), vertical buoyancy production (VBP), and dissipation (DISS) from simulation CI-1 (panel a) and SI-1 (panel b), averaged over days 4-6. Terms are defined as in (35). Also shown is minus the $E B F_{s}$ (gray dashed-dot line), and the scaling for the dissipation (black dashed line) given in equation (47). Panel $\mathrm{c}$ shows a comparison between the scaling for the depth-integrated dissipation rate and the depth-integrated geostrophic shear production plus the buoyancy production across all simulations. The squared correlation coefficient is shown in the plot title. Note that here these terms are evaluated using the approximate form given by (46), ie. $G S P_{s}=-\left\langle v^{\prime} w^{\prime}\right\rangle N_{\infty}^{2} \theta / f\left(1+S_{\infty}^{2}\right)$ and $V B P_{s}=\left\langle w^{\prime} b^{\prime}\right\rangle /\left(1+S_{\infty}^{2}\right)$. The simulation with $V_{\infty}=0.2 \mathrm{~m} \mathrm{~s}^{-1}$ is off the scale shown on this plot, however it also closely follows the 1-1 line. 

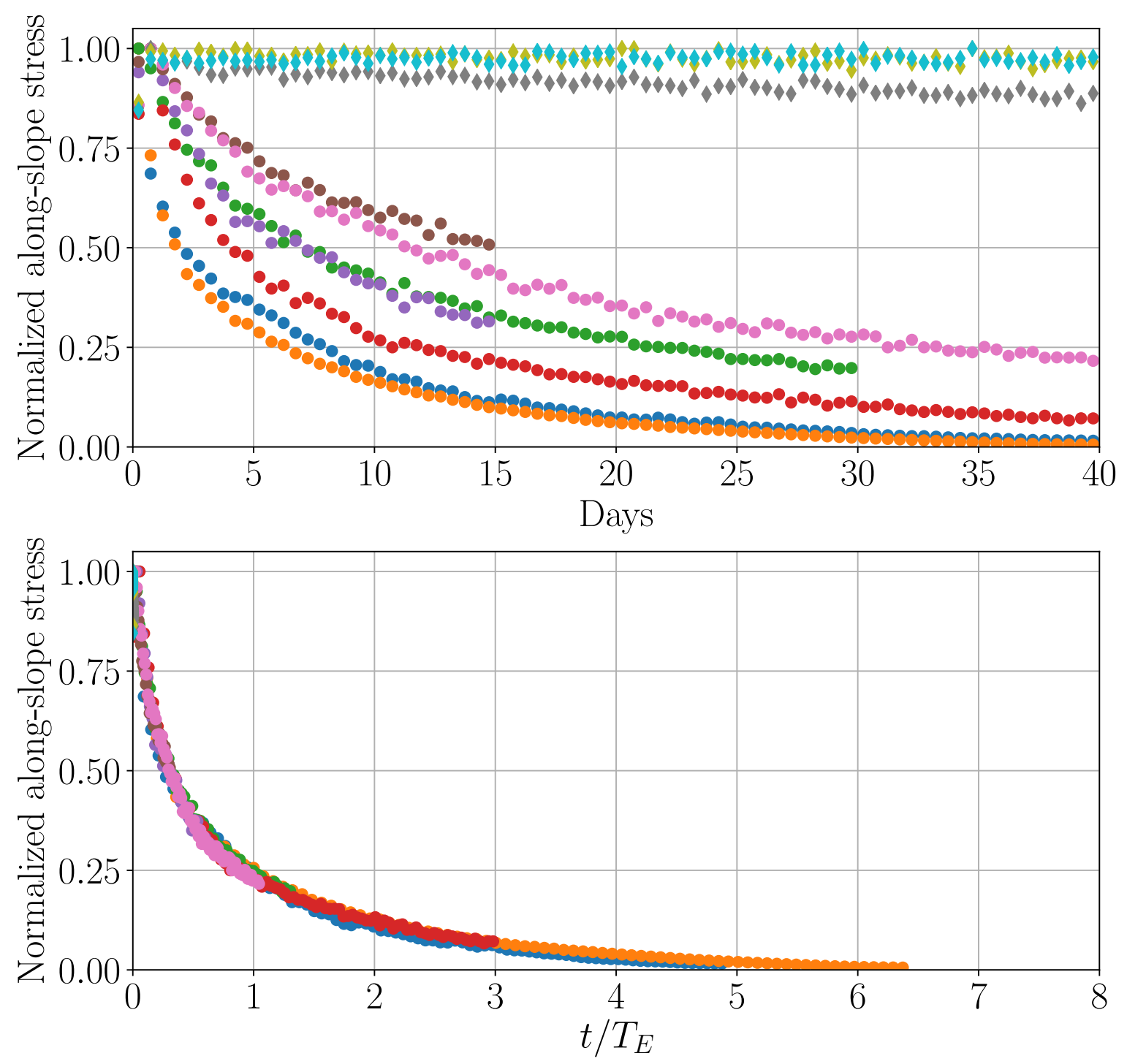

FIG. 16. Evolution of the average along-slope bottom stress, $\left\langle\tau^{y}\right\rangle$, for all simulations (table 1). The top panel shows the evolution of the stress as a function of time. The bottom panel shows the stress evolution with time normalized by the Ekman adjustment timescale, which collapses all simulations to a single curve (similar to the results for 1D simulations shown in Brink and Lentz 2010). Simulations with active SI/CI (circular markers) are normalized using the timescale (49), and simulations where convection dominates (diamond markers) are normalized using (50). In both plots the stress is averaged over 12 hour periods, and normalized by the maximum value for each simulation. 